\title{
On Motion and Force Controllability of Precision Grasps with Hands Actuated by Soft Synergies
}

\author{
D. Prattichizzo Member, IEEE, M. Malvezzi Member, IEEE, \\ M. Gabiccini Member, IEEE, and A. Bicchi Fellow, IEEE
}

\begin{abstract}
To adapt to many different objects and tasks, hands are very complex systems with many degrees of freedom, sensors and actuators. In robotics, such complexity comes at the cost of size and weight of the hardware of devices, but it strongly affects also the ease of their programming. A possible approach to simplification consists in coupling some of the degrees of freedom, thus affording a reduction of the number of effective inputs, and eventually leading to more efficient, simpler and reliable designs. Such coupling can be at the software level, to achieve faster, more intuitive programmability; or at the hardware level, through either rigid or compliant physical couplings between joints.

Physical coupling between actuators and simplification of control through the reduction of independent inputs is also an often-reported interpretation of human hand movement data, where studies have demonstrated that few "postural synergies" explain most of the variance in hand configurations used for grasping different objects. Together with beneficial simplifications, the reduction of the number of independent inputs to a few coupled motions or "synergies" has also an impact on the ability of the hand to dexterously controlling grasp forces and in-hand manipulation. In this paper, through the analysis of a quasi-static model, grasp structural properties related to contact force controllability and object manipulability are defined. The controllable internal forces and motions of the grasped object are related to the actuated inputs: the paper investigates to what extent a hand with many joints can exploit postural synergies to control force and motion of the grasped object.
\end{abstract}

\section{INTRODUCTION}

Robotic hands have many degrees of freedom distributed among several kinematic chains, the fingers. The complexity of the mechanical design is needed to adapt hands to the

D. Prattichizzo is with Dept. of Information Engineering and Mathematical Science, University of Siena, Siena, Italy and Dept. of Advanced Robotics, IIT - Istituto Italiano di Tecnologia, Genova, Italy

M. Malvezzi are with Dept. of Information Engineering and Mathematical Science, University of Siena, 53100 Siena, Italy

M. Gabiccini is with Dept. of Advanced Robotics, IIT - Istituto Italiano di Tecnologia, Genova, Italy, Research Center "E. Piaggio", University of Pisa, Pisa, Italy, and Dept. of Civil and Industrial Engineering, University of Pisa, Italy

A. Bicchi is with Dept. of Advanced Robotics, IIT - Istituto Italiano di Tecnologia, Genova, Italy and Research Center "E. Piaggio", University of Pisa, Pisa, Italy

The authors wish to thank Edoardo Farnioli for his suggestions in the development of the mathematical model. Authors wish furthermore to gratefully acknowledge Marco Santello for the inspiring discussions and for providing experimental data and parameters. This work has been partially supported by the European Commission with the Collaborative Project no. 248587, "THE Hand Embodied", within the FP7-ICT-2009-4-2-1 program "Cognitive Systems and Robotics". many kinds of tasks required in unstructured environments. Roboticists over the years have attempted to imitate the human hand in terms of dexterity and adaption capabilities. Some remarkable example of robotic hand design are the UTAH/MIT hand [28], the DLR hand II [11], the Shadow hand [31]. One of the main issues in designing and controlling robotic hands is that a large number of motors is needed to fully actuate the degrees of freedom but this comes at the cost of size, complexity and weight of the device. This disadvantage could be overtaken if robotic hands were actuated and controlled by a reduced number of inputs, thus resulting more efficient, simpler and reliable than their fully actuated alternatives, as shown in [4], [6], [9].

A promising direction in the design of robotic hands focuses on two key principles: underactuation and passive mechanical adaptation. Underactuation in robotics [8] essentially refers to systems that have more degrees of freedom than actuators. More specifically, in this type of system the Degrees of Freedom DoF are more than the Degrees of Actuation DoA [7]. In grasping and manipulation tasks the unactuated joints often have elastic elements [8], [20], [38], however there are also underactuated hands without elastic elements, e.g. the solution proposed in [19]). If elastic elements are present in non-actuated hand joints we should consider these joints as uncontrollable or passively driven instead of unactuated. The presence of passively actuated joints allows the hand to self-adapt to the surface in a simple and robust way [29], [6]. A quasi-static model of underactuated compliant robotic hands is described in [15]. Grasp properties with underactuated hands and in particular grasp stiffness are analysed in [34]. An anthropomorphic underactuated robotic hand with $15 \mathrm{DoF}$ and a single actuator is described in [27].

A simplified control seems to inspire also biological systems and in particular motor control of human hands, which share with robotic ones the large number of degrees of freedom. Studies in neuroscience [45], [46] demonstrated that a limited set of input variables, named postural synergies, are able to describe most of the variance in hand movements and configurations in grasping tasks. Recently, these studies on human hands inspired new researches on design and control strategies for robotic hands whose main issue is to achieve a trade-off between simplicity, gained through synergy based control, and versatility [10], [16]. In [16] and later in [17] the synergy concept has been applied to control different hand models: a simple gripper, the Barrett hand, the DLR 
hand, the Robonaut hand and the human hand model. In [10] authors proposed a robotic hand design able to match postural synergies mechanically coupling motion of the single joints. Postural synergies in robotic hands allow to control the whole device, with $n_{q}$ joints, through a lower dimension set of actions $n_{z} \leq n_{q}$ : indicating with $\dot{q}$ hand joint velocities, the following relationship can be defined

$$
\dot{q}=S \dot{z}
$$

where $S$ is the synergy matrix and $\dot{z}$ represents synergy velocities. Columns of the matrix of synergies $S \in \Re^{n_{q} \times n_{z}}$ represent the so-called postural synergies, also referred as eigengrasps in the literature, e.g. in [16], in other terms the joint velocities that are obtained acting on each single synergy $\dot{z}_{i}$.

In human hands the synergies can be evaluated analysing measures of hand postures, for instance performing a Principal Component Analysis (PCA) of hand postures during grasping operations in which hand configuration is experimentally measured, as described in [45]. In robotic hands, where a mechanical coupling between joints postural synergies is present, synergies can be derived from the kinematic analysis of joint couplings and constraints. In the artificial, hand synergies can be introduced also at the control level: in this case the coupling between hand joints is managed by the hand control system. In [47] an impedance control for multifingered robotic hands based on the definition of postural synergies was proposed. It was implemented on the DLR hand II [11], whose postural synergies were defined performing a PCA analysis on a wide database of grasp configurations. In [26] a procedure (based on the task-object space) to map human hand synergies on robotic hands, even with a kinematic structure very dissimilar from the anthropomorphic one, is proposed and discussed. In [22] the postural synergies configuration subspace of the UBH (University of Bologna Hand) [30] are evaluated: this study is based on the kinematic structure of the robotic hand and on the taxonomy of the grasp of common objects.

Intuitively, reducing the number of control inputs, from $n_{q}$ actuated joints to $n_{z}$ synergies, may reduce the dimension of the force and motion controllability subspaces thus compromising the dexterity of the given robotic grasp. However, this is not true in general and strongly depends on the column space of synergy matrix $S$. Some of the main questions to answer when interpreting the motion and force control in the light of synergies are: how many synergies have to be involved in a given grasp? which are the contact forces which result to be controllable when acting on synergies instead of each single actuator, independently? is a synergy based actuation of the robotic hand sufficient to guarantee a stable and efficient grasp? what kind of force feedback information is needed to implement the feedback controller based on synergy?

In this paper we tried to find some answer to these questions, extending to synergy controlled hands previous results described in [2], [3], [42].

Differently from other approaches where the actual joint variables is a linear combination of synergies [10], [16], in this paper we define the postural synergies as a joint displacement aggregation corresponding to a reduced dimension representation of hand movements according to a compliant model of joint torques.

Compliance is one of the most important aspects to consider for characterizing the grasp of a robotic hand on an object or on a tool, especially when fine manipulation or high precision is required, e.g. in assembling components. In the analysis of grasping, in general, different compliance sources have to be taken into account: contact stiffness, due for example to fingertip elasticity, actuator stiffness, given by the position control static gain, structural compliance, due to the mechanical deformation of hand elements (joints, drive cables, links, etc.) $[18]$.

A preliminary version of the study of hands with postural synergies was presented in [43]. With respect to that paper, the present one adds details to the grasp model, including terms that were not considered in [43], e.g. geometrical stiffness terms coming from hand Jacobian derivatives [13], [18], [40], and provides a complete solution to the quasi-static model, defining the mapping matrices between the input reference systems and grasp configuration and forces. The evaluation of rigid body motion subspace has been simplified with respect to [43], using results coming from the quasi-static solution. Furthermore, more examples are provided, including an anthropomorphic hand model.

In cases where phalanges have a contact surface, during grasp and manipulation operations it is very common that some of the contacts are rolling [35], [39]. However, since the attention in this paper is focused on the definition of structural grasp properties related on the presence of a limited number of actuators, for the sake of simplicity the study is limited to precision grasps with point contact and friction [44], and the rolling in contact is not considered. Rolling contact modeling is important in the dynamic evolution simulation of grasp as presented in [25]: however, in a quasi-static contest it has effect only in the definition of geometrical stiffness terms [12].

The paper is organized as follows: Section II introduces the main definitions and equations necessary in grasp analysis. Section III describes the contact forces and object motions controllable by the input synergies. Section IV discusses the properties of force and rigid body motion subspaces. Section V shows the results described in the preceding sections with some numerical examples, relative to hands with increasing complexity: a simple gripper, a robotic hand with kinematics similar to the Barrett hand and an anthropomorphic hand. Finally, Section VI concludes the paper. In the Appendix the details of some mathematical steps are provided.

\section{MOdELING HANDS WITH SYNERGIES}

Consider a robotic hand grasping an object as in Fig. 1. Let $\{N\}$ represent the inertial frame fixed in the workspace and let frame $\{B\}$ be fixed to the object. Let $u \in \Re^{n_{d}}$ denote the vector describing the position and orientation of $\{B\}$ relative to $\{N\}, n_{d}=3$ for planar systems, $n_{d}=6$ for spatial systems, $\xi \in \Re^{n_{d}}$ the object twist, and $w \in \Re^{n_{d}}$ the wrench applied to the object, all expressed with respect to $\{B\}$. 


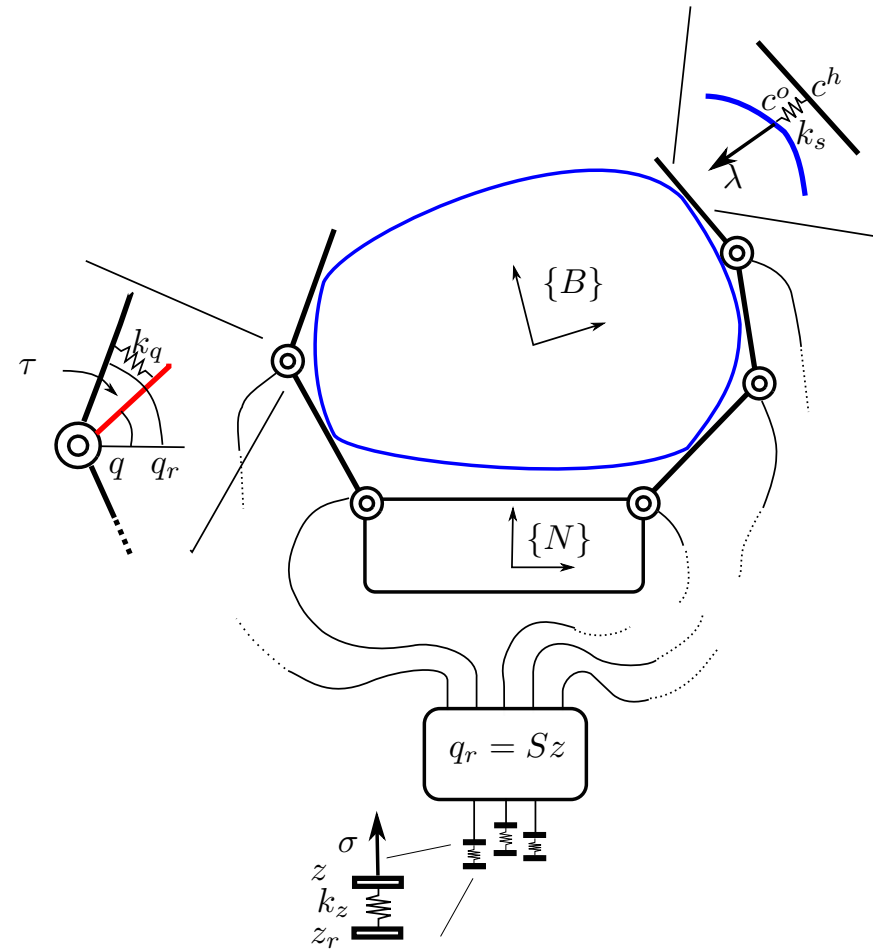

Fig. 1: Hand-object grasp with postural synergies: main quantities.

Let $n_{c}$ be the number of contact points, let $\left\{C_{i}^{h}\right\}$ be the reference frame on the i-th contact point, connected to the hand, and $\left\{C_{i}^{o}\right\}$ the corresponding reference frame connected to the object. Let $\hat{c}_{i}^{h}, \hat{c}_{i}^{o} \in \Re^{n_{d}}$ denote the vector describing the position and orientation of $\left\{C_{i}^{h}\right\}$ and $\left\{C_{i}^{o}\right\}$, respectively, relative to $\{B\}$.

Let $w_{c_{i}^{h}}^{c_{i}^{o}} \in \Re^{n_{d}}$ be the wrench that the hand exerts on the object on the $i$-th contact point, whose components are expressed with respect to $\left\{C_{i}^{o}\right\}$.

A suitable contact model is introduced to define contact constraints and forces [44]: for each contact $i$, the contact force vector $\lambda_{i} \in F C_{i} \subset \Re^{l_{i}}$, is defined, in which $l_{i}$ depends on the contact type and $F C_{i}$ represents the subspace of allowable contact forces. For example, for a point contact with friction model, $l_{i}=3$ and $F C_{i}$ is the so-called friction cone, defined as

$$
F C_{i}=\lambda_{i} \in \Re^{l_{i}}: \sqrt{\lambda_{i, 1}^{2}+\lambda_{i, 2}^{2}} \leq \mu \lambda_{i, 3}
$$

where $\lambda_{i, 1}$ and $\lambda_{i, 2}$ are the contact force components orthogonal to the contact point normal direction, $\lambda_{i, 3}$ represents the normal contact force, and $\mu$ is the friction coefficient [37]. The object static equilibrium equation can be expressed with respect to the object reference frame $\{B\}$ as

$$
w+G \lambda=0
$$

in which $\lambda=\left[\lambda_{1}^{\mathrm{T}}, \cdots, \lambda_{n_{c}}^{\mathrm{T}}\right]^{\mathrm{T}}, \lambda \in \Re^{n_{l}}$, where $n_{l}=\sum_{i=1}^{n_{c}} l_{i}$, and $G \in \Re^{n_{d} \times n_{l}}$ is the Grasp matrix [37].

The general solution of eq. (2), assuming that $w$ is in the column space of $G, \mathcal{R}(G)$, is $\lambda=-G^{+} w+A \chi$, where $G^{+}$is a generic right-inverse of the grasp matrix and $A \in \mathbb{R}^{n_{l} \times n_{h}}$ is a matrix whose columns form a basis of the nullspace of $G$, $\mathcal{N}(G)$, and the vector $\chi \in \mathbb{R}^{n_{h}}$ parametrizes the homogeneous part of the solution. The term $A \chi$ represents the solution to eq. (2) when no external load $w$ is applied, and is usually referred to as internal forces. For general grasp kinematics, and in particular in hands with few actuators, controlling internal forces is not straightforward since the number of internal forces directions, i.e. the dimension of the subspace $\mathcal{N}(G)$, turns out to be larger than the number of controlled joint actions [2], [3].

According to the defined contact model, we can highlight the constrained components of the relative motion in two vectors $v_{c^{o}}^{c^{o}}, v_{c^{h}}^{c^{o}} \in \Re^{n_{l}}$. A linear relationship exists between the contact twist components constrained by the contact model and the object twist, i.e. $v_{c^{o}}^{c^{o}}=G^{\mathrm{T}} \xi$. Similarly we can select from the vectors $\hat{c}_{i}^{o}$ and $\hat{c}_{i}^{h}$ the components $c_{i}^{o}$ and $c_{i}^{h}$, constrained by the contact model and collect them in the vectors $c^{o}=\left[c_{1}^{o}, \cdots, c_{n_{c}}^{o}\right]$ and $c^{h}=\left[c_{1}^{h}, \cdots, c_{n_{c}}^{h}\right]$. We can furthermore approximate the contact frame variation as a function of object configuration variation as

$$
\Delta c^{o}=G^{\mathrm{T}} \Delta u
$$

Let $q=\left[\begin{array}{lll}q_{1} & \cdots & q_{n_{q}}\end{array}\right]^{\mathrm{T}} \in \Re^{n_{q}}$ define the vector of joint displacements. The components of contact point twists on the hand, constrained by the contact model, and expressed with respect to $\left\{C_{i}^{o}\right\}$ reference frames, can be evaluated as a function of hand joint velocities as $v_{c^{h}}^{c^{o}}=J \dot{q}$, in which $J \in \Re^{n_{l} \times n_{q}}$ represents the hand Jacobian matrix. The contact frame displacement can be expressed as a function of joint variation as

$$
\Delta c^{h}=J \Delta q
$$

Considering a generic equilibrium configuration of the hand, the contact forces are balanced by the joint action $\tau \in \Re^{n_{q}}$, i.e.

$$
\tau=J^{\mathrm{T}} \lambda
$$

More details on $G$ and $J$ matrices evaluation of can be found in [37] and are summarized in the Appendix for the reader's convenience.

The hand forward kinematic analysis allows to find a relationship between the synergy value $z$ and the reference joint value $q_{r}$, that can be different from the actual one $q$, since a compliant actuation model is adopted for the hand joints. In the most general case, we can suppose the forward kinematic relationship to be nonlinear

$$
q_{r}=f_{z}(z)
$$

where $f_{z}: \Re^{n_{z}} \rightarrow \Re^{n_{q}}$ represents the kinematic map. More in general, the hand can be represented as a mechanism with $n_{z}$ degrees of freedom, in which the variables $z \in \Re^{n_{z}}$ represent the Lagrangian coordinates adopted as a minimum representation of hand configuration, while $q_{r}$ represent a redundant configuration representation, adopted in this case to simplify the management of the contact with the grasped object. Standard analysis tools for mechanism kinematics can be adopted to define the function $f_{z}$ representing the direct kinematic relationship between redundant configuration 
representation and Lagrangian coordinates $z$ [21], [48]. More practically, the function $f_{z}$ can be determined by the analysis of the mechanical structure of the hand, or evaluated by means of data analysis procedures. For instance, in [45], the synergies for the human hand were evaluated performing the Principal Component Analysis on a set of experimental measures of hand postures, the same technique was adopted in [47] to evaluate the synergies for the DLR-II robotic hand. The kinematic relationship defined in eq. (6) can be differentiated in order to express the joint displacement variation with respect to a initial reference condition, as a function of the synergy variation:

$$
\Delta q_{r}=S \Delta z
$$

where $S=\frac{\partial f_{z}}{\partial z} \in \Re^{n_{q} \times n_{z}}$ is the synergy matrix, that in the more general case depends on hand configuration and then on $z$. Finally, if we consider the hand synergy actuation, in an equilibrium configuration the following relationship between joint torques $\tau$ and synergy generalized forces $\sigma$ holds

$$
\sigma=S^{\mathrm{T}} \tau
$$

Often in robotic hands, and particularly in underactuated ones, compliance could be significant, furthermore, the introduction of compliance allows to solve the indeterminacy of the static problem [2]. According to the definition of grasp matrix and hand Jacobian matrix previously introduced in eq. (3) and (4), a variation of contact force can be expressed as

$$
\Delta \lambda=K_{s}\left(\Delta c^{h}-\Delta c^{o}\right)=K_{s}\left(J \Delta q-G^{\mathrm{T}} \Delta u\right)
$$

where $K_{s} \in \Re^{n_{l} \times n_{l}}$ is the contact compliance matrix, symmetric and positive definite.

As outlined in [18], often the structural stiffness of the links and the controllable servo compliance of the joints have the same order of magnitude of the contact stiffness. A variation of joint torques with respect to a reference initial condition can then be expressed as

$$
\Delta \tau=K_{q}\left(\Delta q_{r}-\Delta q\right)
$$

where $K_{q} \in \Re^{n_{q} \times n_{q}}$ is the joint stiffness matrix, symmetric and positive definite.

Also for the synergies, we assume a compliant model, defined as compliant postural synergies, or soft synergies previously introduced in [5], [23], [24]. According to this compliant model, a variation of the synergy actuation forces can be evaluated as

$$
\Delta \sigma=K_{z}\left(\Delta z_{r}-\Delta z\right)
$$

where $K_{z} \in \Re^{n_{z} \times n_{z}}$ is a symmetric and positive definite matrix that defines the synergy stiffness, and $\Delta z_{r}$ represents the a variation of the synergy reference value.

In the following, we furthermore indicate with $C_{s}, C_{q}$, and $C_{z}$ the compliance matrices, corresponding to stiffness matrices $K_{s}, K_{q}$, and $K_{z}$, respectively, i.e. $C_{s}=K_{s}^{-1}$, and so on.

\section{FORCES AND OBJECT DISPLACEMENTS CONTROLLED} BY SYNERGIES

\section{A. Quasi-static linearized system equations}

Let us consider an equilibrium configuration where an object with an external wrench $w_{0}$ is grasped by a hand whose synergy reference values are $z_{r, 0}$ and the corresponding joint displacements are $q_{0}$. The contact forces in this reference equilibrium are $\lambda_{0}$. Starting from this equilibrium configuration, we consider a small variation of the input synergy reference values $\Delta z_{r}$, which leads to an actual variation of the postural synergies $\Delta z$, to a variation of the joint displacement $\Delta q$ and a variation of contact forces $\Delta \lambda$ for the new equilibrium configuration of the quasi-static model.

Since this work studies the effect on the grasp due to changes of the postural synergies, which play the role of controlled variables, no other actions are considered on the grasp, and we suppose that the object wrench $w_{0}$ is kept constant. If the system is asymptotically stable, after the superimposition of the input variation, it will tend to a new equilibrium configuration [37], [42].

If the new equilibrium configuration is sufficiently near to the reference one, we can assume that the system can be locally linearised.

Let us then consider the equilibrium equations described in the preceding section in the new equilibrium configuration. In eq. (2), the grasp matrix is constant if rolling motion is not considered, and if the object equilibrium equation is expressed with respect to $\{B\}$ reference frame. The object equilibrium in the new configuration, with the same external wrench, can be described by the following equation

$$
0=-G \Delta \lambda
$$

We observe that $\Delta \lambda \in \mathcal{N}(G)$, that is the variation of contact force due to a variation of reference synergy input belongs to the internal force subspace. As discussed in the introduction, for the sake of simplicity this study is limited to precision grasp with point contact and friction [44], and the rolling in contact is not considered. If it was introduced in the model, the variation of contact points during surface rolling should be taken into account by considering also $G$ matrix variation in the linearization of eq. (2), as presented e.g. in [25].

Let us then consider the hand equilibrium equation, according to eq. (5). It is worth to note that the hand Jacobian matrix depends on both the hand joint configuration $q$ and on object displacement vector $u$, i.e. $J=J(q, u)$. The joint torque variation $\Delta \tau$ can be then expressed as

$$
\Delta \tau=J^{\mathrm{T}} \Delta \lambda+K_{J, q} \Delta q+K_{J, u} \Delta u
$$

where $K_{J, q} \in \Re^{n_{q} \times n_{q}}$ and $K_{J, u} \in \Re^{n_{q} \times n_{d}}$ represent the derivatives of hand Jacobian matrix with respect to $q$ and $u$ respectively, evaluated in the reference equilibrium configuration

$$
K_{J, q}=\frac{\partial\left(J^{\mathrm{T}} \lambda_{0}\right)}{\partial q}, \quad K_{J, u}=\frac{\partial\left(J^{\mathrm{T}} \lambda_{0}\right)}{\partial u}
$$

It is worth observing that, even if $K_{J, q}$ and $K_{J, u}$ elements are dimensionally a stiffness, these terms do not represent 
physical stiffness elements, but rather they take account for the dependency of the hand Jacobian on the grasp configuration. For this reason, they are usually referred to as geometric terms. Furthermore, even if $K_{J, q}$ is square, it is in general non symmetric [14]. Both matrices depend on the initial contact force $\lambda_{0}$. In [12], it was proved that these terms are necessary to obtain a conservative congruence transformation from joint torques to workspace wrench.

Finally, concerning the relationship between synergy actions and joint torques, from eq. (8), and recalling that in the more general case the synergy matrix is not constant and depends on the synergy value, we can express the variation $\Delta \sigma$ as follows:

$$
\Delta \sigma=S^{\mathrm{T}} \Delta \tau+K_{S, z} \Delta z
$$

where

$$
K_{S, z}=\frac{\partial S \tau_{0}}{\partial z}
$$

Matrix $K_{S, z} \in \Re^{n_{z} \times n_{z}}$ elements are dimensionally a ratio between a generalized force and a generalized displacement, and depends on the initial torque value $\tau_{0}$.

We can summarize equilibrium conditions described by eq. (12). (13), (15), the constitutive conditions described by eq. (9), (10), (11), and the congruence condition described by eq. (7), in the following linear system

$$
A \Delta x=\Delta y
$$

where $A$ matrix is square and is defined as follows

$$
A=\left[\begin{array}{cccccc}
0 & 0 & G & 0 & 0 & 0 \\
K_{J, u} & K_{J, q} & J^{\mathrm{T}} & -I & 0 & 0 \\
0 & 0 & 0 & S^{\mathrm{T}} & -I & K_{S, z} \\
K_{s} G^{\mathrm{T}} & K_{s} J & I & 0 & 0 & 0 \\
0 & K_{q} & 0 & I & 0 & -K_{q} S \\
0 & 0 & 0 & 0 & I & K_{z}
\end{array}\right]
$$

while $\Delta x$ (unknown terms) and $\Delta y$ (input vector) are defined as

$$
\Delta x=\left[\begin{array}{c}
\Delta u \\
\Delta q \\
\Delta \lambda \\
\Delta \tau \\
\Delta \sigma \\
\Delta z
\end{array}\right] \quad \Delta y=\left[\begin{array}{c}
0 \\
0 \\
0 \\
0 \\
0 \\
K_{z} \Delta z_{r}
\end{array}\right]
$$

The solution of this linear system allows to find a mapping between the input controlled variable, i.e. the synergy reference variation $\Delta z_{r}$, and the output variables. In this paper, in particular, we are interested in the study of internal force variation $\Delta \lambda$, object motion $\Delta u$ and hand configuration variation $\Delta q$.

Here we summarize the main results and input/output relationships that will be necessary to discuss the controllability of internal forces and object motion in the following section. The solution of the linear system is detailed in the Appendix.
By defining the following matrices

$$
\begin{aligned}
G_{K}^{+} & =K_{s} G^{\mathrm{T}}\left(G K_{s} G^{\mathrm{T}}\right)^{-1} \\
P_{q} & =\left(I-G_{K}^{+} G\right) K_{s} J \\
V_{q} & =\left(G K_{s} G^{\mathrm{T}}\right)^{-1} G K_{s} J \\
U_{q} & =\left(J^{\mathrm{T}} P_{q}+K_{J, q}+K_{J, u} V_{q}\right) \\
X & =\left(U_{q}+K_{q}\right)^{-1} K_{q} \\
Z & =\left(S^{\mathrm{T}} U_{q} X S+K_{S, z}\right) \\
Y & =\left(Z+K_{z}\right)^{-1} K_{z}
\end{aligned}
$$

the solution of the system described in eq. (17) can be expressed as

$$
\begin{aligned}
\Delta z & =Y \Delta z_{r} \\
\Delta \sigma & =Z Y \Delta z_{r} \\
\Delta q & =X S Y \Delta z_{r} \\
\Delta \tau & =U_{q} X S Y \Delta z_{r} \\
\Delta u & =V_{q} X S Y \Delta z_{r} \\
\Delta \lambda & =P_{q} X S Y \Delta z_{r}
\end{aligned}
$$

Eq. (20), (22) and (23) can be simplified as

$$
\begin{aligned}
\Delta q & =X S Y \Delta z_{r} \\
\Delta u & =V \Delta z_{r} \\
\Delta \lambda & =P \Delta z_{r}
\end{aligned}
$$

where $Q=X S Y, P=P_{q} X S$, and $V=V_{q} X S Y$ are matrices mapping synergy references values to joint configuration, contact force and object configuration variations, respectively.

Remark 1: Starting from a reference configuration and acting on synergies $\Delta z_{r}$, the joint displacements depends both on the synergy matrix $S$ and on the whole system compliance. When the hand is making contact with an object or with the environment, $\Delta q \neq S \Delta z_{r}$.

If the synergy actuation is perfectly stiff, i.e. if $C_{z}=0$, it is easy to show that $Y=I$ and thus $\Delta z=\Delta z_{r}$. Furthermore, if the links are perfectly stiff and the joint control gains are infinite, i.e. $C_{q}=0$, it results that $X=I$ and $\Delta q=S \Delta z$. Summarizing, in case of $C_{z}=0$ and $C_{q}=0$ one gets a simplified version of eq. (24)

$$
\Delta q=S \Delta z=S \Delta z_{r}
$$

which is similar to the definition of synergy control given in [10], [16].

\section{B. Controllable internal forces}

In eq. (26), $\Delta \lambda$ corresponds to the contact force variation obtained by applying a variation on the reference synergy variables $\Delta z_{r}$, without modifying the external wrench $w_{0}$. These contact forces can be referred to as controllable internal forces: controllable, since they can be modified by acting on $\Delta z_{r}$, internal because they do not involve a variation in the external wrench applied on the object [2].

The control of internal forces is paramount in robotic grasping [44]. It allows to steer the contact forces to satisfy 
the constraints imposed by friction at the contacts, thus guaranteeing not to loose adhesion with the object which would compromise the whole grasp.

From eq. (26), we can define a basis matrix $E_{s}$ for the subspace of controllable internal forces by postural synergies as

$$
\mathcal{R}\left(E_{s}\right)=\mathcal{R}(P)
$$

All internal forces controllable by synergy actions can then be parametrized through a free vector $\alpha$, i.e.

$$
\Delta \lambda=E_{s} \alpha
$$

\section{Controllable rigid-body object motions and hand joint redundant motions}

Eq. (25) shows how the object displacements $\Delta u$ are controlled from one equilibrium configuration to another by small synergy variations $\Delta z_{r}$. Among all the possible motions of the grasped objects, rigid-body motions are paramount since they do not involve visco-elastic deformations in the contact points.

A rigid-body motion is characterized by a null variation of the contact force $\Delta \lambda$, and then, from eq. (9), the following constraint equations hold

$$
J \Delta q-G^{\mathrm{T}} \Delta u=0,
$$

which relates joint displacements and object displacement.

We then need to evaluate which object rigid-body motions, complying with eq. (29), are controllable acting on synergies. We observe that the synergy reference values that modify hand and object configuration without modifying the contact force values, from eq. (26), are a solution of the homogeneous system

$$
P \Delta z_{r}=0
$$

in other terms rigid body motions are generated by reference synergy variations $\Delta z_{r h}$ that belong to the $P$ matrix nullspace

$$
\Delta z_{r h} \in \mathcal{N}(P)
$$

The corresponding object displacement, $\Delta u_{h}$, according to eq. (25) is given by

$$
\Delta u_{h}=V \Delta z_{r h}
$$

Furthermore, the corresponding hand configuration variation $\Delta q_{h}$, according to eq. (24) is described by the vector

$$
\Delta q_{h}=Q \Delta z_{r h}
$$

We observe that the synergy reference values defined in the subspace

$$
\Delta z_{r r} \in(\mathcal{N}(P) \cap \mathcal{N}(V))
$$

i.e. the solutions of the homogeneous contact force problem defined in eq. (30) that belongs to the nullspace of $V$ matrix, do not produce contact force variation, neither object motion: they then modify hand configuration without modifying object conditions and can be referred to as hand redundant motions. The corresponding hand configuration variation is

$$
\Delta q_{r r}=Q \Delta z_{r r}
$$

It is clear from eq. (29) that hand and object configurations variations that do not involve contact force modifications can be evaluated by computing

$$
\mathcal{N}\left[J \quad-G^{\mathrm{T}}\right]
$$

as described, for instance, in [41]. A matrix $\Gamma$ can then be defined, whose columns form a basis of such subspace. Under the hypothesis that the object motion is not indeterminate [44], i.e. $\mathcal{N}\left(G^{\mathrm{T}}\right) \neq 0$, i.e. the object is completely restrained by contacts, matrix $\Gamma$ can be expressed as

$$
\Gamma=\mathcal{N}\left(\left[\begin{array}{ll}
J & -G^{T}
\end{array}\right]\right)=\left[\begin{array}{cc}
\Gamma_{q r} & \Gamma_{q c} \\
0 & \Gamma_{u c q}
\end{array}\right]
$$

where $\Gamma_{q r}$ is a basis matrix of the subspace of redundant motions $\mathcal{N}(J)$, and $\Gamma_{q c}$ and $\Gamma_{u c q}$ are conformal partitions of a complementary basis matrix. The image spaces of $\Gamma_{q c}$ and $\Gamma_{u c q}$ consist of coordinated rigid-body motions of the mechanism, for the hand configuration and the object position and orientation, respectively.

The description of rigid body motion in eq. (37) does not take into account the synergy actuation system and then the solution found with this method could be infeasible acting on synergies. The rigid body motions compatible with object equilibrium equation and reachable acting on the synergy reference values are given by

$$
\Delta u_{r b} \in \mathcal{R}\left(\Gamma_{u c s}\right)=\left(\mathcal{R}(V) \cap \mathcal{R}\left(\Gamma_{u c q}\right)\right)
$$

It is worth to note that rigid-body motions of the object are not all the possible motions of the object controlled by synergies as in (25), since the subspace of all synergy controlled object motion $\mathcal{R}(V)$ also contains motions due to deformations of elastic elements in the model. Summarizing, all rigid-body displacements of the object can be parametrized through a free vector $\beta$ as

$$
\Delta u_{h}=\Gamma_{u c s} \beta
$$

Similarly the description of hand joint redundant motions obtained from eq. (37) do not take into account the synergy actuation system and then also in this case the solution found with this method could be unfeasible acting on the synergies. The hand joint redundant motions $\Delta q_{r r}$, reachable by acting on the synergy reference values $\Delta z_{r}$ belong to the subspace $\Gamma_{q r s}$ defined as

$$
\Delta q_{r r} \in \mathcal{R}\left(\Gamma_{q r s}\right)=\left(\mathcal{R}(Q) \cap \mathcal{R}\left(\Gamma_{q r}\right)\right)
$$

Summarizing, all hand redundant motions can be parametrized through a free vector $\gamma$ as

$$
\Delta q_{r r}=\Gamma_{q r s} \gamma
$$

\section{INTERNAL FORCES AND RIGID-BODY MOTION CONTROL}

In grasps by hands controlled with few synergies it is possible that not all the object motions and contact forces result controllable by synergistic actions. According to eq. (38), desired quasi-static rigid-body object motions $\Delta u_{\text {des }}$ can be performed if they remain within subspace $\mathcal{R}\left(\Gamma_{u c s}\right)$ and 
analogously, according to eq. (26) and (28), arbitrary quasistatic contact force displacements $\Delta \lambda_{\text {des }}$ can be performed if they evolve within subspace $\mathcal{R}\left(E_{s}\right)$ defined in eq. (28). It is worth noting that with the results obtained up to here, we can arbitrarily control motions in $\mathcal{R}\left(\Gamma_{u c s}\right)$ or contact forces in $\mathcal{R}\left(E_{s}\right)$ but we are not guaranteed that in coupled motion and force control, we can jointly but independently control two vectors lying on these subspaces.

In grasping, however, due to the presence of unilateral, conic contact constraints, task specifications can not be given disjointly in terms of either object positions or contact forces. Therefore conditions $\Delta u_{\text {des }} \in \mathcal{R}\left(\Gamma_{u c s}\right)$ and $\Delta \lambda_{\text {des }} \in \mathcal{R}\left(E_{s}\right)$ are only necessary, but no longer sufficient, for joint control of object motions and contact forces. Moreover, specifications of jointly controllable object motions and contact forces may not exhaust the control capabilities of synergy actions for the given grasp due to the presence of synergy redundancy.

Our goal is to define a set of controlled outputs for a grasp with synergies that is guaranteed to be feasible with synergy actions, that fully exploits the control inputs, and that is convenient for the specification of the tasks. The first requirement implies that the output vector of forces and motions can be controlled by synergies, the second that controlled output vector has the same dimension $n_{z}$ of the synergy vector $z_{r}$ and the third that the output vector consider the typical approach of a grasping task:

- contact forces that can be controlled so as to avoid violation of contact constraints;

- object trajectories that can be accommodated for by the grasp with synergies;

- reconfiguration of limbs in the presence of redundancy in synergies.

The following theorem proposes a set of outputs for grasps with synergies.

Theorem 1: Under the technical assumption that the grasp is not indeterminate $\left(\mathcal{N}\left(G^{\mathrm{T}}\right)=0\right)$, consider the quasi-static model of any grasp with synergies described in (25) and (26). It is always possible to control, jointly but independently, the controllable internal forces, the rigid-body object motions and redundancy with the synergy displacement $\Delta z_{r}$ control input. In the general case, since the system is compliant, a variation in the contact forces cause a displacement of the grasped object. Therefore, in general, contact force variation and object displacement are related. This theorem states that, if we have a sufficient number of DoF, we can use them to control independently internal forces and rigid-body object motions. Thus, in this context, jointly and independently means that contact forces and object motions are not related each other and can be independently controlled acting on the available inputs. Algebraically, this corresponds to state that for any $\alpha$, $\beta$ and $\gamma$, there always exists a $\Delta z_{r}$ solving the linear system of equations

$$
\left[\begin{array}{l}
E_{s} \alpha \\
\Gamma_{u c s} \beta \\
\Gamma_{q r s} \gamma
\end{array}\right]=\left[\begin{array}{l}
P \\
V \\
Q
\end{array}\right] \Delta z_{r}
$$

where $\Gamma_{u c s}$ and $\Gamma_{q r}$ have been defined in eq. (37), and $E_{s}$ has been defined in eq. (28).
Moreover, solution for $\Delta z_{r}$ is unique and the number of synergies $n_{z}$ is equal to the sum of the dimensions of the controlled output subspaces:

$$
n_{z}=\#\left(E_{s}\right)+\#\left(\Gamma_{u c s}\right)+\#\left(\Gamma_{q r s}\right)
$$

\section{Proof:}

The theorem and the proof were originally presented in [43], however it has been summarized here for the readers' convenience, since several additional considerations on hand model, discussed in the previous sections, have been proposed with respect to that work.

Linear system (40) can be rewritten as

$$
\left[\begin{array}{c}
\alpha \\
\beta \\
\gamma
\end{array}\right]=\left[\begin{array}{c}
E_{s}^{+} P \\
\Gamma_{u c s}^{+} V \\
\Gamma_{q r s}^{+} Q
\end{array}\right] \Delta z_{r}
$$

where $B^{+}=\left(B^{\mathrm{T}} B\right)^{-1} B^{\mathrm{T}}$ denotes the pseudoinverse of a basis matrix $B$.

The linear system in eq. (42) is square if the number $\#\left(\Gamma_{u c s}\right)+\#\left(E_{s}\right)+\#\left(\Gamma_{z r}\right)=n_{z}$ where $\#(N)$ denotes the number of colums of matrix $N$. This can be proved by observing that, since $\Gamma_{z r}, \Gamma_{u c s}$, and $E_{s}$ are full column rank by definition, observing that $\# E_{s}=\# \mathcal{R}(P)$, according to eq. (28), and $\# \mathcal{R}\left(\Gamma_{u c s}\right)+\# \mathcal{R}\left(\Gamma_{q r}\right)=\# \mathcal{N}(P)$, according to eq. (38) and (39).

To complete the proof it suffices to show that coefficient matrix in (42) is full row rank which is equivalent to prove that

$$
\mathcal{N}\left(\left[\begin{array}{c}
E_{s}^{+} P \\
\Gamma_{u c s}^{+} V \\
\Gamma_{z r}^{+} Q
\end{array}\right]^{\mathrm{T}}\right)=\{0\} .
$$

Each block of the matrix in the equation above is full column rank, in fact:

- $E_{s}$ is a basis for $\mathcal{R}(P)$ (cf. eq. (28));

- $\mathcal{R}\left(\Gamma_{u c s}\right) \subseteq \mathcal{R}(V)$, directly from eq. (38) and is a basis for object rigid body motions;

- $\Gamma_{z r} \subseteq \mathcal{R}(Q)$ from eq. (39) and is a basis for hand redundant motions.

Hence, to prove eq. (43) it is sufficient to show that the raw spaces of the three blocks are also mutually linearly independent and this directly follows from the definitions of internal forces, rigid body object motions and redundant hand motions in eq. (28), (38), and (39).

Remark 2: The result in eq. (41) deals with dimensions of subspaces and is numerical in nature. It states a very basic structural property of grasp analysis with postural synergies: if $n_{z}$ control inputs are available, one cannot control, jointly and independently, more than $n_{z}$ variables among internal forces, object motion directions and kinematic redundancy.

Remark 3: The result in eq. (40) deals with grasp control with postural synergies. When the mechanical structure is complex, with many joints, but the control inputs are few, it is not easy to understand which synergy one needs to activate to accomplish a given tasks. The solution of linear system in eq. (40) allows to simply compute the control variables, the synergy references, according to the task to be performed. 


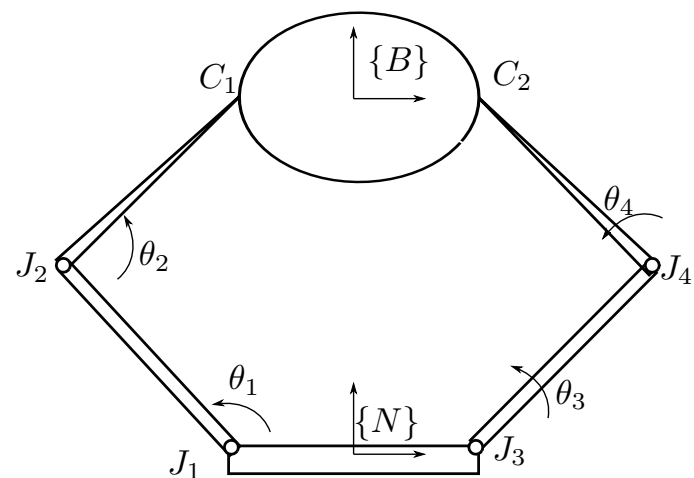

Fig. 2: A four DoF planar gripper grasping an object with two contact points.

These results are useful also to find the minimal design requirements in terms of number of synergies to be used to accomplish the given task.

It is worth emphasizing that the motions of the object considered in this paper are those performed with respect to the palm of the hand. In other terms, we are considering fine motion control of grasped objects more than large displacements, which can be performed by moving the wrist of the arm.

Finally, note that results presented in this paper are still valid for fully actuated robotic hands provided the matrix $S$ is substituted with the identity matrix.

\section{NUMERICAL EXAMPLES}

The numerical simulations presented in this section were performed using Syngrasp [33], a set of Matlab functions devoted to the simulation and analysis of the main properties of grasps performed with synergy actuated hands.

\section{A. Simple gripper}

As a first example, let us consider a simple gripper like the one shown in Fig. 2. The gripper is planar and has two fingers, each of which is composed of two phalanges with the same lengths: the gripper has then $4 \mathrm{DoF}$ total. Let $J_{1}, \cdots, J_{4}$ be the traces of the joint axes on the gripper plane, and let $\theta_{1}, \cdots, \theta_{4}$ be the joint angles. The gripper is grasping an object with its fingertips. The contact points are $C_{1}$ and $C_{2}$, the origin of the local object reference frame is on the mean point of $C_{1} C_{2}$ segment, and the local $x$ axis is parallel to $C_{1} C_{2}$ direction. The contact model assumed in this test is the Point Contact With Friction (PCWF) [37], often indicated also as Hard Finger (HF) [44]. Then, each contact force has two components, i.e. $\lambda_{1}=\left[\begin{array}{ll}\lambda_{1 x} & \lambda_{1 y}\end{array}\right]^{\mathrm{T}}$ and $\lambda_{2}=\left[\begin{array}{ll}\lambda_{2 x} & \lambda_{2 y}\end{array}\right]^{\mathrm{T}}$, defined w.r.t. the object fixed reference frame. The object displacement is defined w.r.t. the base reference system by the vector $u=\left[\begin{array}{llll}u_{x} & u_{y} & \phi\end{array}\right]^{\mathrm{T}}$, where $\phi$ represents the angle between the local and the base $x$-axes. We consider a reference configuration in which the external load $w_{0}$ is balanced by the contact forces $\lambda_{01}$ and $\lambda_{02}$, applied at the points $C_{1}$ and $C_{2}$ respectively: the contact vector is then defined as $\lambda_{0}=\left[\begin{array}{llll}\lambda_{01 x} & \lambda_{01 y} & \lambda_{02 x} & \lambda_{02 y}\end{array}\right]^{\mathrm{T}}$.
Indicating with $a$ the length of the finger phalanges, the hand Jacobian matrix is defined as follows

$$
J=\left[\begin{array}{cccc}
J_{1,1} & J_{1,2} & 0 & 0 \\
J_{2,1} & J_{2,2} & 0 & 0 \\
0 & 0 & J_{3,3} & J_{3,4} \\
0 & 0 & J_{4,3} & J_{4,4}
\end{array}\right]
$$

in which the matrix terms can be expressed as:

$$
\begin{aligned}
& J_{1,1}=s \phi\left(a c_{12}+a c_{1}\right)-c \phi\left(a s_{12}+a s_{1}\right) \\
& J_{1,2}=a c_{12} s \phi-a s_{12} c \phi \\
& J_{2,1}=s \phi\left(a s_{12}+a s_{1}\right)+c \phi\left(a c_{12}+a c_{1}\right) \\
& J_{2,2}=a s_{12} s \phi+a c_{12} c \phi \\
& J_{3,3}=s \phi\left(a c_{34}+a c_{3}\right)-c \phi\left(a s_{34}+a s_{3}\right) \\
& J_{3,4}=a c_{34} s \phi-a s_{34} c \phi \\
& J_{4,3}=s \phi\left(a s_{34}+a s_{3}\right)+c \phi\left(a c_{34}+a c_{3}\right) \\
& J_{4,4}=a s_{34} s \phi+a c_{34} c \phi
\end{aligned}
$$

with $s_{1}=\sin \theta_{1}, c_{1}=\cos \theta_{1}, s_{12}=\sin \left(\theta_{1}+\theta_{2}\right)$, and so on. The grasp matrix is given by

$$
G=\left[\begin{array}{cccc}
1 & 0 & 1 & 0 \\
0 & 1 & 0 & 1 \\
0 & -r & 0 & r
\end{array}\right]
$$

where $r$ represents object radius, i.e. the distance between each contact point and the object frame origin.

The geometric terms, that express the variation of $J$ matrix, with respect to $q$ and $u$, are considered by defining the matrices $K_{J, q}$ and $K_{J, u}$ as outlined in eq. (14). The matrix $K_{J, q}$ is given by

$$
K_{J, q}=\left[\begin{array}{cccc}
k_{J, q, 1,1} & k_{J, q, 1,1} & 0 & 0 \\
k_{J, q, 2,1} & k_{J, q, 2,2} & 0 & 0 \\
0 & 0 & k_{J, q, 3,3} & k_{J, q, 3,4} \\
0 & 0 & k_{J, q, 4,3} & k_{J, q, 4,4}
\end{array}\right]
$$

in which the matrix terms can be expressed as:

$$
\begin{aligned}
& k_{J, q, 1,1}=-\lambda_{01, x}\left(c \phi\left(a c_{12}+a c_{1}\right)+s \phi\left(a s_{12}+a s_{1}\right)\right) \\
&-\lambda_{01, y}\left(a c_{12} c \phi+a s_{12} s \phi\right) \\
& k_{J, q, 1,2}=-\lambda_{01, x}\left(c \phi a c_{12}+s \phi a s_{12}\right)-\lambda_{01, y}\left(a c_{12} c \phi+a s_{12} s \phi\right) \\
& k_{J, q, 2,1}= \lambda_{01, x}\left(s \phi\left(a c_{12}+a c_{1}\right)-c \phi\left(a s_{12}+a s_{1}\right)\right) \\
&+\lambda_{01, y}\left(a c_{12} s \phi-a s_{12} s \phi\right) \\
& k_{J, q, 2,2}= \lambda_{01, x}\left(a c_{12} s \phi-a s_{12} c \phi\right)+\lambda_{01, y}\left(a c_{12} s \phi-a s_{12} c \phi\right) \\
& k_{J, q, 3,3}=-\lambda_{02, x}\left(c \phi\left(a c_{34}+a c_{3}\right)+s \phi\left(a s_{34}+a s_{3}\right)\right) \\
& k_{J, q, 3,4}=-\lambda_{02, y}\left(a c_{34} c \phi+a s_{34} s \phi\right) \\
& k_{J, q, 4,3}= \lambda_{02, x}\left(c \phi a c_{34}+s \phi a s_{34}\right)-\lambda_{02, y}\left(a c_{34} c \phi+a s_{34} s \phi\right) \\
& k_{J, q, 4,4}=+\lambda_{02, y}\left(a c_{34} s \phi-a s_{34} s \phi\right) \\
& k_{02, x}\left(a c_{34} s \phi-a s_{34} c \phi\right)+\lambda_{02, y}\left(a c_{34} s \phi-a s_{34} c \phi\right)
\end{aligned}
$$

while the matrix that expresses the hand Jacobian derivatives with respect to object displacement is given by

$$
K_{J, u}=\left[\begin{array}{ccc}
0 & 0 & k_{J, u, 1,3} \\
0 & 0 & k_{J, u, 2,3} \\
0 & 0 & k_{J, u, 3,3} \\
0 & 0 & k_{J, u, 4,3}
\end{array}\right]
$$

in which the matrix terms can be expressed as:

$$
\begin{aligned}
k_{J, u, 1,3}= & \lambda_{01, x}\left(c \phi\left(a c_{12}+a c_{1}\right)+s \phi\left(a s_{12}+a s_{1}\right)\right) \\
& +\lambda_{01, y}\left(a c_{12} c \phi+a s_{12} s \phi\right) \\
k_{J, u, 2,3}= & -\lambda_{01, x}\left(s \phi\left(a c_{12}+a c_{1}\right)-c \phi\left(a s_{12}+a s_{1}\right)\right) \\
k_{J, u, 3,3}= & -\lambda_{01, y}\left(a c_{12} s \phi-a s_{12} c \phi\right) \\
& \lambda_{02, x}\left(\mathbf{c} \phi\left(a c_{34}+a c_{3}\right)+s \phi\left(a s_{34}+a s_{3}\right)\right) \\
k_{J, u, 4,3}= & \lambda_{02, y}\left(a c_{34} c \phi+a s_{34} s \phi\right) \\
& \left.\left.+a s_{3}\right)\right)+\lambda_{02, y}\left(a c_{34}\right)+s \phi\left(a s_{34}\right) \\
&
\end{aligned}
$$


In the numerical simulations that follow, we assume that the reference configuration is described by these quantities

- $\theta_{1}=\frac{3}{4} \pi \mathrm{rad}, \theta_{2}=-\frac{\pi}{2} \mathrm{rad}, \theta_{3}=\frac{\pi}{4} \mathrm{rad}, \theta_{4}=\frac{\pi}{2} \mathrm{rad}$,

- $w_{0}=0 \mathrm{~N}, \lambda_{01}=[1,0]^{\mathrm{T}} \mathrm{N}, \lambda_{02}=[-1,0]^{\mathrm{T}} \mathrm{N}$,

- $a=0.3 \mathrm{~m}$.

The stiffness matrices are $K_{s}=k_{s} I_{4,4}, K_{q}=k_{q} I_{4,4}$, where $k_{s}=1000 \mathrm{~N} / \mathrm{m}, k_{q}=1000 \mathrm{Nm} / \mathrm{rad}$, and $I_{4,4}$ represents the four dimensional diagonal matrix. In this simple example, we suppose to control each joint independently, which implies $S=I_{4,4}$, and we ignore the synergy stiffness.

According to eq. (26), in the above described reference configuration, we obtain the following matrix $P$ that maps input joint references to contact forces:

$$
P=\left[\begin{array}{rrrr}
-67.44 & -33.95 & 67.25 & 33.58 \\
0.00 & 0.00 & 0.00 & 0.00 \\
67.44 & 33.95 & -67.25 & -33.58 \\
0.00 & 0.00 & 0.00 & 0.00
\end{array}\right]
$$

It easy to verify that in this case $\#(P)=1$ and a basis of $P$ image is

$$
E=\left[\begin{array}{r}
-0.71 \\
0.00 \\
0.71 \\
0.00
\end{array}\right]
$$

that consists of two forces whose direction is along the $x$ axis of the object reference frame, with the same moduli and opposite signs. Consequently $\# \mathcal{N}(P)=3$ and thus the rigid body object motions compatible with the contact constraints, that do not involve contact force variations can be described as a generic translation and rotation of the object on the plane, as shown in Fig. 3.

A basis of the rigid-body motions compatible with hand kinematics and actuation system can be calculated also as the intersection between the object motions that do not produce contact force variations, i.e. that belong to $\mathcal{N}\left(\left[\begin{array}{ll}J & -G^{\mathrm{T}}\end{array}\right]\right)$, and the object motion that do no produce external load variation, i.e. that belong to $\mathcal{N}(G)$; in the reference configuration previously described, from eq. (37) we have

$$
\Gamma=\mathcal{N}\left(\left[\begin{array}{ll}
J & -G^{\mathrm{T}}
\end{array}\right]\right)=\left[\begin{array}{rrr}
-0.77 & 0.00 & 0.00 \\
0.26 & 0.76 & 0.00 \\
-0.51 & 0.44 & 0.38 \\
-0.26 & -0.11 & -0.76 \\
0.09 & -0.05 & 0.00 \\
0.02 & 0.03 & 0.03 \\
0.00 & -0.46 & 0.53
\end{array}\right]
$$

and then the object body motions that do not modify contact forces are in the subspace defined by

$$
\Gamma_{u c q}=\left[\begin{array}{rrr}
0.09 & -0.05 & 0.00 \\
0.02 & 0.03 & 0.03 \\
0.00 & -0.46 & 0.53
\end{array}\right]
$$

It is easy to note that this subspace is equivalent to those evaluated as $\mathcal{N}(P)$ and corresponds to a generic translation and rotation of the object in the plane.

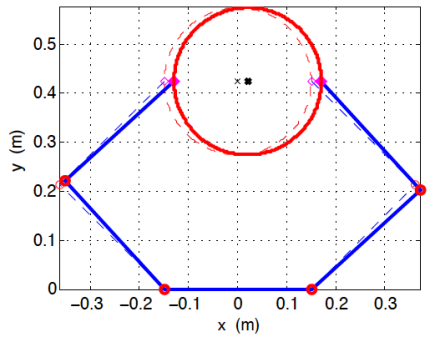

a)

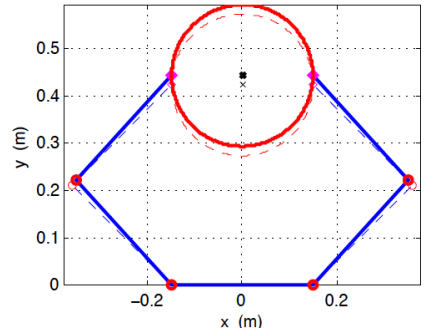

b)

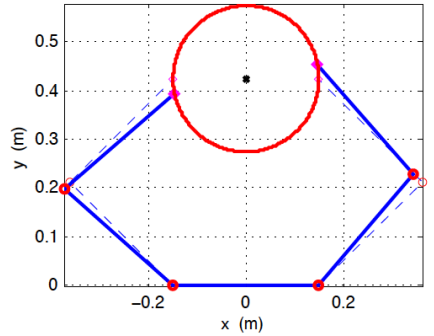

c)

Fig. 3: Four DoF planar gripper example: a basis for object rigid body motions. The rigid body motion subspace has dimension three. The dashed lines represent the initial reference position, the solid ones represent the modified configuration. a) translation in the $x$ direction, $\mathrm{b}$ ) translation in the $y$ direction, c) object rotation.

\section{B. Barrett hand}

Results on the motion and force control of grasps with synergies have been then applied to a robotic hand whose kinematics was inspired by the Barrett Hand [1], shown in Figure 4: it is a three finger, eight-axis mechanical hand, in which each finger has two joints. One of the fingers, referred to as 1 , is stationary, while the other two can spread synchronously up to 180 degrees about the palm. Although there are eight axes, the hand is actuated by four motors: each finger has an actuated proximal link and a coupled distal link that moves at a fixed rate with the inner link. An additional motor control the synchronous spread of the two fingers about the palm. In the Barrett hand, a clutch mechanism allows the outer link to continue to move even if the inner link motion is obstructed, however this feature has not been considered in the present analysis.

Let us define $\theta_{i, 1}(i=1, . ., 3)$ the rotation of the inner link with respect to the palm, $\theta_{i, 2}(i=1, . ., 3)$ the rotation of the outer link with respect to the inner one and $\theta_{i, 0} \quad(i=2,3)$ the spread of the two fingers about the palm. Thus the configuration vector can be defined as: $q=\left[\theta_{1,1}, \theta_{1,2}, \theta_{2,0}, \theta_{2,1}, \theta_{2,2}, \theta_{3,0}, \theta_{3,1}, \theta_{3,2}\right]^{T}$. The Denavit Hartenberg parameters for the Barrett hand are summarized in Table I a). In the numerical simulations, we assumed $a_{1}=a_{2}=0.05 \mathrm{~m}$.

The mechanical couplings between the joints are expressed 


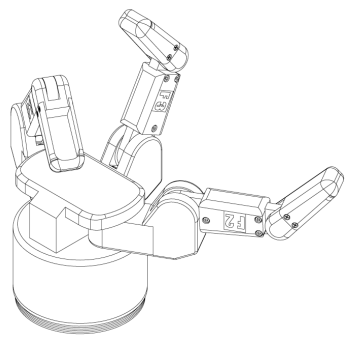

a)

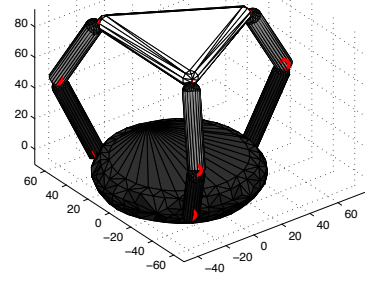

b)
Fig. 4: The Barrett Hand a) the robotic hand, b) the mathematical model representation, including the hand links, joints, base, grasped object and contact points

\begin{tabular}{|c|c|c|c|c|}
\hline link & $\alpha_{i}$ & $a_{i}$ & $\theta_{i}$ & $d_{i}$ \\
\hline \hline finger 1 & & & & \\
\hline 1 & 0 & $a_{1}$ & $\theta_{1,1}$ & 0 \\
2 & 0 & $a_{2}$ & $\theta_{1,2}$ & 0 \\
\hline finger 2 & & & & \\
\hline 1 & 0 & 0 & $\theta_{2,0}$ & 0 \\
2 & $\pi / 2$ & $a_{1}$ & $\theta_{2,1}$ & 0 \\
3 & 0 & $a_{2}$ & $\theta_{2,2}$ & 0 \\
\hline finger 3 & & & & \\
\hline 1 & 0 & 0 & $\theta_{3,0}$ & 0 \\
2 & $\pi / 2$ & $a_{1}$ & $\theta_{3,1}$ & 0 \\
3 & 0 & $a_{2}$ & $\theta_{3,2}$ & 0 \\
\hline
\end{tabular}

\begin{tabular}{|c|c|}
\hline$\theta_{i}$ & value $(\mathrm{rad})$ \\
\hline$\theta_{1,1}$ & $\pi / 3$ \\
$\theta_{1,2}$ & $\pi / 3$ \\
$\theta_{2,0}$ & $2 / 3 \pi$ \\
$\theta_{2,1}$ & $\pi / 3$ \\
$\theta_{2,2}$ & $\pi / 3$ \\
$\theta_{3,0}$ & $-2 / 3 \pi$ \\
$\theta_{3,1}$ & $\pi / 3$ \\
$\theta_{3,2}$ & $\pi / 3$ \\
\hline
\end{tabular}

a)

b)

TABLE I: Parameters for the Barrett hand example: a) Denavit Hartenberg parameters, b) joint angles in the reference configuration.

by the following relationships:

$$
\begin{aligned}
\theta_{2,0} & =-\theta_{3,0}=z_{1} \\
\theta_{i, 2} & =\alpha_{i} \theta_{i, 1}=z_{i+1} \quad i=1, . ., 3
\end{aligned}
$$

where $\alpha_{i}$ represents the ratio between the outer and the inner angle for the i-th link. In the numerical simulation described above, we assumed $\alpha_{i}=1$. The joint angles are controlled acting on four parameters, collected in the vector $z=\left[z_{1}, \ldots, z_{4}\right]^{T}$. Accordingly, the synergy matrix can be defined as

$$
S=\left[\begin{array}{cccc}
0 & 1 & 0 & 0 \\
0 & \alpha_{1} & 0 & 0 \\
1 & 0 & 0 & 0 \\
0 & 0 & 1 & 0 \\
0 & 0 & \alpha_{2} & 0 \\
-1 & 0 & 0 & 0 \\
0 & 0 & 0 & 1 \\
0 & 0 & 0 & \alpha_{3}
\end{array}\right]
$$

In this example, the contact stiffness matrix has been chosen as $K_{s}=k_{s} I_{9}$, where $k_{s}=1000 \mathrm{~N} / \mathrm{m}$ and $I_{9}$ is the $9 \times 9$ identity matrix. The joint stiffness matrix has been chosen as $K_{q}=k_{q} I_{8}$, where $k_{q}=1000 \mathrm{Nm} / \mathrm{rad}$ and $I_{8}$ is the 8 -dimensional identity matrix. Finally, the synergy stiffness matrix has been chosen as $K_{z}=k_{z} I_{8}$, where $k_{z}=1000$ $\mathrm{Nm} / \mathrm{rad}$ and $I_{8}$ is the $8 \times 8$ identity matrix. The initial contact

\begin{tabular}{|r||c|c|c|}
\hline & $E$ & $\Gamma_{u c s}$ & $\Gamma_{z r}$ \\
\hline synergies (4 inputs) & 3 & 1 & 0 \\
fully actuated (8 joints) & 3 & 2 & 3 \\
\hline
\end{tabular}

TABLE II: Barrett Hand: controllable internal forces and allowable movements for the synergy actuated (4 degrees of freedom) and fully actuated ( 8 degrees of freedom) hand.

force $\lambda_{0}$ has been considered zero, so that, the components of the geometric terms $K_{J, q}$ and $K_{J, u}$ vanish.

Reference values for the hand joints are summarized in Table I b). The contact points between the hand and the grasped object were located on the three finger-tips and the normal directions at the contact points have been thought as oriented towards the center of the object. Hard Finger (HF) contact model has been considered in this example.

Hand Jacobian matrix $J$ dimensions are $9 \times 8$, matrix $G$ dimensions are $6 \times 9$, and $\mathcal{N}(G)$ dimension is 3 . According to the previously described analysis, the dimension of the controllable internal forces and object motions have been evaluated with both the hypothesis that the hand is controlled with the four actuators, and considering the case when all the eight joints are actuated. Table II summarizes the obtained results, in particular the dimensions of the controllable internal forces, rigid-body motions and hand redundancy subspaces. We can observe that, in all the cases, the sum between the dimensions of $E_{s}, \Gamma_{u c s}$ and $\Gamma_{z r}$ is equal to the number of synergies that is to the number of actuated joints.

In particular, for the synergy actuated case, the following values were obtained for matrices $P$ and $V$ :

$$
\begin{aligned}
& P=\left[\begin{array}{rrrr}
0.17 & 0.42 & 0.04 & 0.04 \\
0 & 0 & 0.28 & -0.28 \\
0 & 0 & 0 & 0 \\
-0.09 & -0.21 & -0.26 & 0.22 \\
0.15 & 0.08 & -0.39 & -0.12 \\
0 & 0 & 0 & 0 \\
-0.09 & -0.21 & 0.22 & -0.26 \\
-0.15 & .08 & 0.12 & 0.39 \\
0 & 0 & 0 & 0
\end{array}\right] \\
& V=\left[\begin{array}{rrrr}
-0.44 & 0.19 & -0.10 & -0.10 \\
0 & 0 & -0.02 & 0.02 \\
0 & 0.01 & 0.01 & 0.01 \\
0 & 0 & -0.05 & 0.05 \\
0.15 & -0.11 & 0.06 & 0.06 \\
0 & 0 & 0.05 & -0.05
\end{array}\right]
\end{aligned}
$$

It is possible to verify that in this case $\#(P)=3$ and a basis of the controllable internal forces is given by:

$$
E_{s}=\left[\begin{array}{rrr}
0.06 & 0.08 & 0.81 \\
-0.46 & 0.35 & 0 \\
0 & 0 & 0 \\
0.37 & -0.34 & -0.41 \\
-0.19 & -0.73 & 0.09 \\
0 & 0 & 0 \\
-0.43 & 0.26 & -0.40 \\
0.65 & 0.39 & -0.09 \\
0 & 0 & 0
\end{array}\right]
$$

Figure 5 shows, for the first three synergies, the hand motion (first row) and the set of internal contact forces generated acting through each single synergy, evaluated by means of equation (26) (second row). Only three synergies have been 

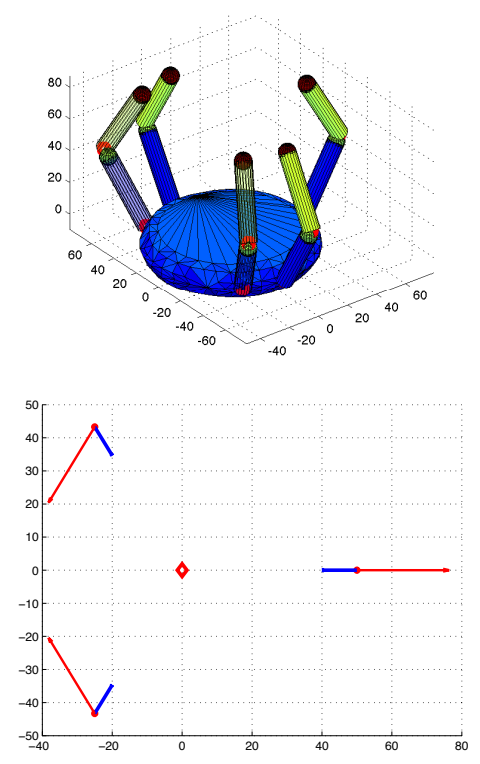
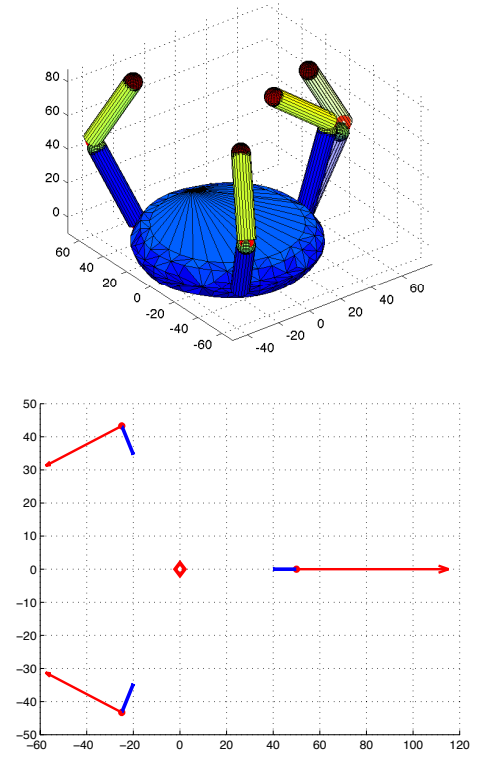
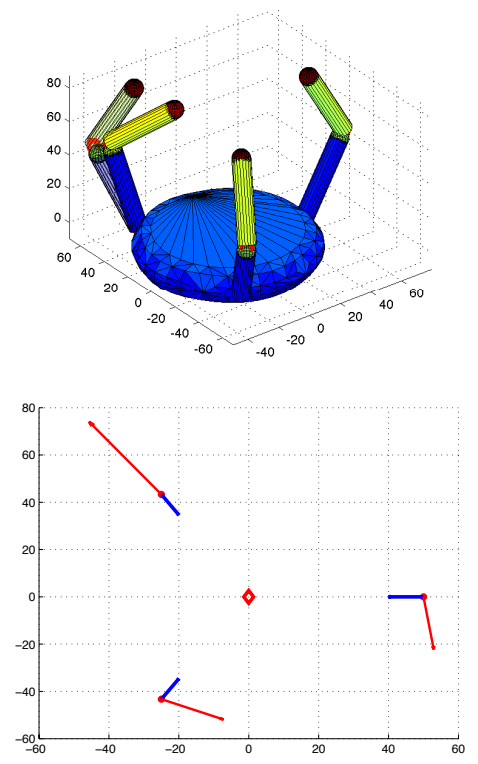

Fig. 5: Synergies in the Barrett hand. First row: hand configuration obtained acting on each synergy; second row: contact points (red dots), object center (red square), contact normal unit vectors (blue arrows) and internal forces (red arrows) generated activating each synergy.

represented since the activation of the fourth synergy is symmetric with respect to the third one.

\section{Application to a human like robotic hand}

The analysis of controllable internal forces and object movements has been applied to a robotic hand with an anthropomorphic kinematic structure, actuated with synergies. The details of the kinematic model are described in [36]. The kinematic model of the anthropomorphic hand considered has globally $20 \mathrm{DoF}$, four degrees of freedom for each finger. In this paper, a tripod grasp has been considered: the object (a cherry) is grasped with the thumb, index and middle. Each of the three fingers touches the object only in its tip. A Hard Finger (HF) contact model has been considered (single point contact with friction). The layout of the hand and the object is shown in Fig. 6.

The contact force and joint vector dimensions are then $n_{l}=$ 9 and $n_{q}=20$ respectively. Thus for the fully actuated hand, grasp matrix and hand Jacobian dimensions are, respectively, $G \in \Re^{6 \times 9}$ and $J \in \Re^{9 \times 20}$. The dimension of internal force subspace is $e=\#(P)=3$, the rank of rigid body motion subspace is $\#\left(\Gamma_{u c}\right)=4$, and the redundancy is $\#\left(\Gamma_{q r}\right)=13$.

In order to reduce the number of controlled joint inputs a synergy based actuation system has been considered. The synergy matrix $S$ is computed such that its columns are the Principal Components (PCs) extracted from the data set presented in [45]. In this work the authors collected a large set of data containing grasping poses from subjects that were asked to shape their hands in order to mime grasps for a large set $(N=57)$ of common objects. Principal Components Analysis (PCA) of this data revealed that the first two principal

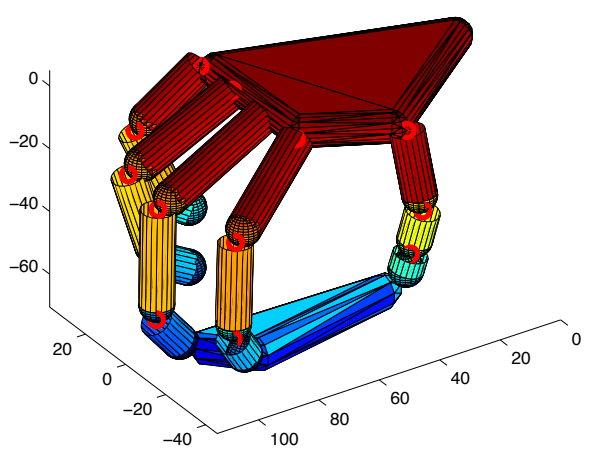

Fig. 6: The human-like hand and the grasped object models in the reference configuration.

components account for more than the $80 \%$ of the variance, suggesting that a satisfying characterization of the recorded data can be obtained using a much lower-dimensional subspace of the hand DoF space. These and similar results seem to suggest that, out of the 20 or more DoFs of a human hand, only a few combinations can be used to shape the hand for basic grasps used in everyday life. The data were obtained using an instrumented glove that measured the configuration of 15 hand joints. From the available experimental data, the PCA returned a $15 \times 15$ matrix whose columns represented the principal components of the data set, ordered in such a way that the first one accounts for the largest possible variance (that is, accounts for as much of the variability in the data as possible), and each succeeding component, in turn, has the highest variance possible under the constraint 
to be orthogonal to (i.e., uncorrelated with) the preceding components. Since the anthropomorphic model we adopted has 20 DoFs, while the measured joints were 15 , we extended with some heuristic considerations, based on human hand anatomy, the PCA results and obtained a complete synergy matrix $S_{\text {tot }} \in \Re^{20 \times 15}$. From this matrix we selected the synergy matrix $S \in \Re^{20 \times n_{z}}$, with $n_{z}$ varying from 1 to 15 , selecting from $S_{t o t}$ the first $n_{z}$ columns. In the preceding section the synergies were modelled through a compliant structure. Also in this case the contact stiffness matrix has been chosen as $K_{s}=k_{s} I_{9}$, where $k_{s}=100 \mathrm{~N} / \mathrm{m}$ and $I_{9}$ is the $9 \times 9$ identity matrix. The joint stiffness matrix has been chosen as $K_{q}=k_{q} I_{20}$, where $k_{q}=1000 \mathrm{Nm} / \mathrm{rad}$ and $I_{20}$ is the $20 \times 20$ identity matrix. Finally, the synergy stiffness matrix has been chosen as $K_{z}=k_{z} I_{n_{z}}$, where $k_{z}=10000 \mathrm{Nm} / \mathrm{rad}$ and $I_{n_{z}}$ is the $n_{z} \times n_{z}$ identity matrix. For the sake of simplicity, the initial contact force $\lambda_{0}$ has been considered null, so that, the components of the geometric terms $K_{J, q}$ and $K_{J, u}$ are null.

The number of engaged synergies has been progressively increased from 1 to 9 , in the order obtained from PCA decomposition of experimental measures [43], [45].

Fig. 7 shows the internal force variation $\Delta \lambda$ and the corresponding object displacement $\Delta u$ obtained activating one synergy once, i. e. $\Delta \lambda_{i}=P \Delta z_{r i}$,

$$
\Delta u_{i}=P \Delta z_{r i}
$$

where $\Delta z_{r i}=[0,0, \ldots, 1, \ldots, 0]^{\mathrm{T}}$.

In order to verify the results presented in the preceding sections, we analysed, for different numbers of engaged synergies, the dimensions of controllable internal forces $e=\#(P)$, rigid body motions $\#\left(\Gamma_{u c}\right)$, and redundant hand motion $\#\left(\Gamma_{r}\right)$. Results in terms of dimensions of controllable forces and movements subspaces, are shown in Tab. III. We observe that, by increasing the number of engaged synergies from 1 to 3 , the number of controllable internal forces increases from 1 to 3 , while no rigid motions are possible. Increasing the number of engaged synergies from 4 to 7 , the dimension of controllable rigid body motions increases from 1 to 4 , while the controllable internal force subspace do not increase any more, since it dimension reached the size of $\mathcal{N}(G)$. Finally, further increasing the number of synergies, either the controllable rigid body motion dimensions fulfill and hand redundant motions appear. From the results summarized in Tab. III it is evident that the maximum dimension of the subspace of controllable object rigid motion, in this application, is $\# \Gamma_{u c s}=4$, therefore it is not possible to fully control the sixdimensional motion of the object without modifying contact forces. In particular, if for instance, only $n_{z}=6$ synergies are activated, $\# \Gamma_{u c s}=3$, and the following basis for object rigid body motions can be found:

$$
\Gamma_{u c s}=\left[\begin{array}{rrr}
-4.11 & 2.76 & 4.48 \\
-10.3 & 3.08 & 10.6 \\
-3.91 & -5.45 & -8.74 \\
-0.11 & -0.12 & -0.39 \\
-0.06 & 0.12 & -0.28 \\
-0.20 & 0.09 & 0.39
\end{array}\right]
$$
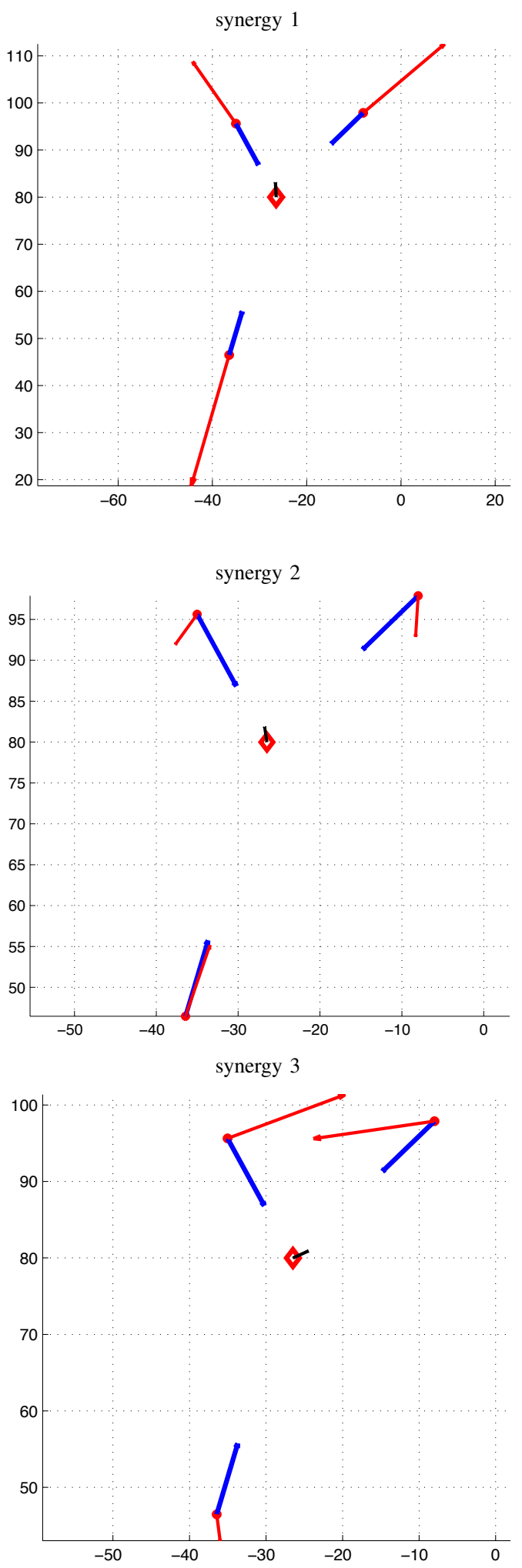

Fig. 7: Forces and movements produced by activating one synergy once. Internal forces (red arrows), contact point unitary vectors (blue arrows), and object displacement (black arrow) induced by the application of one synergy once, projected on the $x y$ plane. The red round dots represent the contact points, the red square dot is the object center. 


\begin{tabular}{|c|c|c|c|}
\hline$n_{z}$ & $\# E$ & $\# \Gamma_{u c s}$ & $\Gamma_{r}$ \\
\hline \hline 1 & 1 & 0 & 0 \\
2 & 2 & 0 & 0 \\
3 & 3 & 0 & 0 \\
4 & 3 & 1 & 0 \\
5 & 3 & 2 & 0 \\
6 & 3 & 3 & 0 \\
7 & 3 & 4 & 0 \\
8 & 3 & 4 & 1 \\
9 & 3 & 4 & 2 \\
\hline
\end{tabular}

TABLE III: Human-like hand: dimension of controllable internal forces $E_{s}$, rigid body motions $\Gamma_{u c}$, redundant motions $\Gamma_{r}$, as a function of the number of activated synergies.

that can be obtained by choosing the reference synergy values from the following base:

$$
\Gamma_{z r}=\left[\begin{array}{rrr}
-0.61 & 0 & 0 \\
-0.27 & -0.93 & 0 \\
-0.57 & 0.18 & 0.12 \\
-0.36 & 0.27 & 0.38 \\
0.31 & -0.15 & 0.70 \\
0.03 & 0.03 & -0.59
\end{array}\right]
$$

If, for instance, we were interested only in the translational part of the motion, without care about rotations, we could evaluate the synergy variation necessary to produce the desired displacement of the object center, $\Delta p_{\text {des }}$, as follows:

$$
\Delta z_{r}=\Gamma_{z r} \Gamma_{u c s, t}^{-1} \Delta p_{d e s}
$$

where $\Gamma_{u c s, t}$ is obtained from the first three rows of matrix $\Gamma_{u c s}$. For example, to obtain a displacement of $1 \mathrm{~mm}$ in the $x$, $y$, and $z$ directions, respectively, without changing the contact forces, the following synergy reference variations have to be commanded

$$
\begin{aligned}
\Delta z_{r x} & =[0.10,-0.63,0.18,0.12,-0.43,0.24,]^{\mathrm{T}} \\
\Delta z_{r y} & =[0,0.27,-0.03,-0.01,0.17,-0.12,]^{\mathrm{T}} \\
\Delta z_{r z} & =[0.05,0.01,0.04,0.02,-0.09,0.05,]^{\mathrm{T}}
\end{aligned}
$$

These examples show how the proposed model can be used to define the synergy input variations necessary to perform a desired task, that could consist of a variation in the contact forces and/or on a displacement of the grasped object with respect to the hand reference frame. Furthermore, the examples show how the model could be used in the design phase of the hand and of its control system, to define the structural properties of the hand synergy system necessary to realize pre-defined tasks.

\section{Conclusions}

In grasping hands with $n_{z}$ postural synergies, a structural relationship exists between the dimension of controllable internal forces and object motion subspaces, and the number of synergy control inputs. In particular, if the hand is actuated controlling the reference values of the synergy vector $z_{r} \in$ $\Re^{n_{z}}$, it is not possible to control, jointly and independently, more than $n_{z}$ variables among internal forces, object motion directions and kinematic redundancy. This paper provides geometric and structural properties of hands actuated by a postural synergy system, that are the fundamentals for the design of control strategies to perform complex manipulation tasks, involving both control of motion and forces, through very few control inputs.

Furthermore, tools for design requirements of complex robotic hands in terms of number of synergies to accomplish manipulation tasks are provided. We believe that providing structural and basic results like the controllability of forces and motions in hand grasps with postural synergies will allow to better understand and exploit the synergies in both robotics and human studies. Some numerical examples, relative to a simple gripper, a three fingered robotic hand with a kinematic structure similar to the the Barrett Hand, and an anthropomorphic hand are shown putting the theory on a test.

\section{APPENDIX}

\section{A: Grasp matrix and hand Jacobian matrix evaluation}

In the following, we describe the evaluation of grasp matrix and hand Jacobian matrix in the three-dimensional case, i.e. for $n_{d}=6$. The simplification for the bi-dimensional case (i.e. $n_{d}=2$ ) is straightforward. Further details and examples can be found, for instance in [37] and in [44]. A detailed explanation of the representation can be found in [32].

Let $g_{b c_{i}^{o}}$ be the vector describing the configuration of frame $\left\{C_{i}^{o}\right\}$ with respect to $\{B\}$, let furthermore $g_{n c_{i}^{h}}$ be the vector describing the configuration of frame $\left\{C_{i}^{h}\right\}$ with respect to $\{N\}$, we use the Product of Exponential (PoE) formula for its parametrization, i.e.

$$
g_{n c_{i}^{h}}=\left(\prod_{k=1}^{m_{i}} e^{\hat{\psi}_{k} q_{k}^{i}}\right) g_{n c_{i}^{h}}(0)
$$

where $\psi_{k} \in \Re^{6}$ is an element of $s e(3)$ and $\hat{\psi}_{k} \in \Re^{4}$ is its homogeneous form, $q_{i}^{k}$ are the exponential coordinates for a local representation of $S E(3)$ and $g_{n c_{i}^{h}}(0)$ is the initial configuration.

Grasp matrix: Let $w_{c_{i}^{h}}^{c_{i}^{o}} \in \Re^{n_{d}}$ be the wrench that the hand exerts on the object on the $i$-th contact point, whose components are expressed with respect to $\left\{C_{i}^{o}\right\}$ reference frame. The object equilibrium, with respect to $\{B\}$ reference system, can be expressed as

$$
w+\sum_{i=1}^{n_{c}} \operatorname{Ad}_{g_{b c_{i}^{o}}}^{-\mathrm{T}} w_{c_{i}^{h}}^{c_{i}^{o}}=0
$$

For each contact point $i$, we can introduce the vector $\lambda_{i} \in$ $F C_{i}$, where $F C_{i}$ is a subspace of dimension $l_{i}$ whose value depends on the type of contact. For each contact point we can define a matrix $H_{i} \in \Re^{n_{d} \times l_{i}}$ that maps the local vector $\lambda_{i}$ onto the contact wrench $w_{c_{i}^{h}}^{c^{o}}$

$$
w_{c_{i}^{h}}^{c_{i}^{o}}=H_{i} \lambda_{i}
$$

The equilibrium equation can be written as

$$
w+\sum_{i=1}^{n_{c}} \operatorname{Ad}_{g_{b c_{i}^{o}}^{-\mathrm{T}}}^{-\mathrm{T}} H_{i} \lambda_{i}=0
$$


The contact vectors can be organized in the vector $\lambda=$ $\left[\lambda_{1}^{\mathrm{T}}, \cdots, \lambda_{n_{c}}^{\mathrm{T}}\right]^{\mathrm{T}} \in \Re^{n_{l}}$, the equilibrium equation can be furthermore simplified as

$$
w+G \lambda=0
$$

where the Grasp matrix $G \in \Re^{n_{d} \times n_{l}}$ is defined as

$$
G=\left[\operatorname{Ad}_{g_{b c_{1}^{o}}}^{-\mathrm{T}} H_{1}, \cdots, \operatorname{Ad}_{g_{b c_{n_{c}}^{o}}^{-\mathrm{T}}}^{-\mathrm{T}} H_{n_{c}}\right]
$$

Let $\xi$ be the twist that describes the $\{B\}$ frame motion with respect to $\{N\}$, expressed with respect to $\{B\}$ reference frame, and let $\xi_{c^{o}}^{c^{o}}$ be the twists of frames $\left\{C_{i}^{o}\right\}$, expressed with respect to $\left\{C_{i}^{o}\right\}$. These twists are related by the following relationship

$$
\xi_{a b}^{c_{i}^{o}}=\operatorname{Ad}_{g_{c_{i}^{o b}}} \xi
$$

Since a contact model has been defined, that constraints some of the relative motion components, we can highlight these components in a vector $v_{c_{i}^{o}}^{c_{i}^{o}} \in \Re^{l_{i}}$, by means of the transpose of the selection matrix previously defined

$$
v_{c_{i}^{o}}^{c_{i}^{o}}=H_{i}^{\mathrm{T}} \xi_{c_{i}^{o}}^{c_{i}^{o}}=H_{i}^{\mathrm{T}} \operatorname{Ad}_{g_{c_{i}^{o} b}} \xi,
$$

All these components can be collected in the vector

$$
v_{c^{o}}^{c^{o}}=\left[v_{c_{1}^{o}}^{c_{1}^{o} \mathrm{~T}}, \cdots, v_{c_{n_{c}}^{o}}^{c_{n_{c}}^{o} \mathrm{~T}}\right]^{\mathrm{T}} \in \Re^{n_{l}}
$$

It is easy to observe that

$$
\begin{aligned}
v_{c^{o}}^{c^{o}} & =\left[\left(H_{1}^{\mathrm{T}} \operatorname{Ad}_{g_{c_{1}^{o} b}}\right)^{\mathrm{T}}, \cdots,\left(H_{n_{c}}^{\mathrm{T}} \operatorname{Ad}_{g_{c_{n_{c}} b}}\right)^{\mathrm{T}}\right]^{\mathrm{T}} \xi \\
& =G^{\mathrm{T}} \xi
\end{aligned}
$$

For the sake of simplicity, in the text, we did not consider the relationship between angular speed and time derivative of angular displacements. When we multiply the above equation by the time $\Delta t$, in the rotational part, $\xi_{a b}^{b} \Delta t$ does not represent a coordinate variation. However, we can define a matrix $T(u) \in \Re^{n_{d} \times n_{d}}$ that allows to express the frame twist as a function of $u$ time derivative, i.e.

$$
\xi=T(u) \dot{u}
$$

by defining the contact frame variation as $\Delta c_{i}^{o}=v_{c_{i}^{o}}^{c_{i}^{o}} \Delta t$ we can write $\Delta c^{o}=\tilde{G}^{\mathrm{T}} \Delta u$, where $\tilde{G}^{\mathrm{T}}=G^{\mathrm{T}} T(u)$. It is worth to note that $G$ matrix elements do not depends on object displacement $u$, while $\tilde{G}$ does, since $T$ depends on $u$.

1) Hand Jacobian matrix: Let $\xi_{n}^{c_{i}^{o}}$ represent the twist of a frame instantaneously superimposed to the frame $\{N\}$ that is fixed with $\left\{C_{i}^{h}\right\}$ with components expressed with respect to $\{N\}$. This twist depends on the joint velocities $\dot{q}^{i}$ as follows

$$
\xi_{n}^{c_{i}^{o}}={ }^{n} J_{i}\left(q^{i}\right) \dot{q}^{i}
$$

where ${ }^{n} J_{i}\left(q^{i}\right)$ is the spatial Jacobian relative to the $i$-th contact point, defined as

$$
{ }^{n} J_{i}\left(q^{i}\right)=\left[\xi_{1}^{i}, \xi_{2}^{\prime i}, \cdots, \xi_{m_{i}}^{\prime i}\right]
$$

with

$$
\xi_{j}^{i i}=\operatorname{Ad}_{g_{1(j-1)}} \xi_{j}^{i}
$$

and

$$
g_{1(j-1)}=\prod_{k=1}^{j-1} e^{\hat{\xi}_{k}^{i} q_{k}^{i}}
$$

Let then $\xi_{c_{i}^{h}}^{c_{i}^{o}}$ the twists of frames $\left\{C_{i}^{h}\right\}$, expressed with respect to $\left\{C_{i}^{o}\right\}$. To find the twist $\xi_{c_{i}^{h}}^{c_{i}^{o}}$ we can use adjoint matrix obtaining

$$
\xi_{c_{i}^{h}}^{c_{i}^{o}}=\operatorname{Ad}_{g_{c_{i}^{o} n}(u)^{n}} J_{i}\left(q^{i}\right) \dot{q}^{i}=\tilde{J}_{i} \dot{q}^{i}
$$

with $\tilde{J}_{i}\left(q^{i}, u\right)=\operatorname{Ad}_{g_{c_{i}^{o} n}(u)}^{n} J_{i}\left(q^{i}\right)$. We can then highlight the velocity components constrained by the contact model in a vector $v_{c_{i}^{h}}^{c_{i}^{o}} \in \Re^{l_{i}}$, by means of the transpose of the selection matrix previously defined

$$
v_{c_{i}^{h}}^{c_{i}^{o}}=H_{i}^{\mathrm{T}} \xi_{c_{1}^{h}}^{c_{i}^{o}}=H_{i}^{\mathrm{T}} \tilde{J}_{i} \dot{q}^{i}
$$

All these components can be collected in the vector

$$
v_{c^{h}}^{c^{o}}=\left[v_{c_{1}^{h}}^{c_{1}^{o} \mathrm{~T}}, \cdots, v_{c_{n_{c}}^{h}}^{c_{n_{c}}^{o}}\right]^{\mathrm{T}} \in \Re^{n_{l}}
$$

Similarly we can collect all the joint variables in a vector

$$
q=\left[q_{1}^{\mathrm{T}}, \ldots, q_{F}^{\mathrm{T}}\right]^{\mathrm{T}} \in \Re^{n_{q}}
$$

The components of the contact point velocities $v_{c^{h}}^{c^{o}}$ are related to the hand joint velocities $\dot{q}$ by the following relationship

$$
v_{c^{h}}^{c^{o}}=J(q, u) \dot{q}
$$

where

$$
J(q, u)=\left[\begin{array}{ccc}
H_{1}^{\mathrm{T}} \tilde{J}_{1} & \cdots & 0 \\
\cdots & \cdots & \cdots \\
\cdots & 0 & H_{n c}^{\mathrm{T}} \tilde{J}_{n c}
\end{array}\right]
$$

is the Hand Jacobian matrix. It is worth to note that, since we expressed the contact twists with respect to the object fixed $\left\{C_{i}^{o}\right\}$ reference frames, $J$ depends both on hand configuration $q$ and on object displacement $u$.

Let the components of the components of contact point twists on the hand, constrained by the contact model, and expressed with respect to $\left\{C_{i}^{o}\right\}$ reference frames, can be evaluated as a function of hand joint velocities as follows

$$
v_{c^{h}}^{c^{o}}=J \dot{q}
$$

in which $J \in \Re^{n_{l} \times n_{q}}$ represents the hand Jacobian matrix. It is worth to note that the hand Jacobian matrix depends on both the hand joint configuration $q$ and on object displacement vector $u$, i.e. $J=J(q, u)$.

\section{B: System solution}

Let us consider the system composed of the equilibrium conditions described by eq. (12). (13), (15), the constitutive conditions described by eq. (9), (10), (11), and the congruence condition described by eq. (7), and summarized in eq. (17).

By substituting eq. (9) in eq. (12), we can express $\Delta u$ as a function of $\Delta q$

$$
\begin{gathered}
G K_{s}\left(J \Delta q-G^{\mathrm{T}} \Delta u\right)=0 \\
G K_{s} J \Delta q=G K_{s} G^{\mathrm{T}} \Delta u=0 \\
\Delta u=\left(G K_{s} G^{\mathrm{T}}\right)^{-1} G K_{s} J \Delta q=V_{q} \Delta q
\end{gathered}
$$

where $V_{q}=\left(G K_{s} G^{\mathrm{T}}\right)^{-1} G K_{s} J$.Let us substitute eq. (46) in eq. (9), we can then express $\Delta \lambda$ as a function of $\Delta q$

$$
\Delta \lambda=K_{s}\left(J \Delta q-G^{\mathrm{T}}\left(G K_{s} G^{\mathrm{T}}\right)^{-1} G K_{s} J \Delta q\right)
$$




$$
\Delta \lambda=\left(I-G_{K}^{+} G\right) K_{s} J \Delta q=P_{q} \Delta q
$$

where $G_{K}^{+}=K_{s} G^{\mathrm{T}}\left(G K_{s} G^{\mathrm{T}}\right)^{-1}$ is the $G$ matrix pseudoinverse, weighted with $K_{s}$ matrix and $P_{q}=\left(I-G_{K}^{+} G\right) K_{s} J$. Let us then consider eq. (13) and substitute $\Delta \lambda$ and $\Delta u$ with eq. (47) and (46), respectively

$$
\Delta \tau=\left(J^{\mathrm{T}} P_{q}+K_{J, q}+K_{J, u} V_{q}\right) \Delta q=U_{q} \Delta q
$$

with $U_{q}=\left(J^{\mathrm{T}} P_{q}+K_{J, q}+K_{J, u} V_{q}\right)$. By substituting eq. (48) in eq. (10) we can express $\Delta q$ as a function of $\Delta q_{r}$

$$
\begin{aligned}
& \Delta \tau=K_{q}\left(\Delta q_{r}-\Delta q\right) \\
& U_{q} \Delta q=K_{q}\left(\Delta q_{r}-\Delta q\right) \\
& \left(U_{q}+K_{q}\right) \Delta q=K_{q} \Delta q_{r} \\
\Delta q= & \left(U_{q}+K_{q}\right)^{-1} K_{q} \Delta q_{r}=X \Delta q_{r}
\end{aligned}
$$

where $X=\left(U_{q}+K_{q}\right)^{-1} K_{q}$. Taking into account eq. (7) we can express $\Delta q$ as a function of $\Delta z$

$$
\Delta q=X S \Delta z
$$

and consequently, by substituting in eq. (48) we obtain

$$
\Delta \tau=U_{q} X S \Delta z
$$

By substituting eq. (51) in eq. (15) we can express $\Delta \sigma$ as a function of $\Delta z$

$$
\begin{gathered}
\Delta \sigma=S^{\mathrm{T}} \Delta \tau+K_{S, z} \Delta z \\
=\left(S^{\mathrm{T}} U_{q} X S+K_{S, z}\right) \Delta z \\
\Delta \sigma=\left(S^{\mathrm{T}} U_{q} X S+K_{S, z}\right) \Delta z=Z \Delta z
\end{gathered}
$$

where $Z=\left(S^{\mathrm{T}} U_{q} X S+K_{S, z}\right)$. Finally, by substituting eq. (52) in eq. (11) we can express $\Delta z$ as a function of $\Delta z_{r}$

$$
\begin{aligned}
& \Delta \sigma=K_{z}\left(\Delta z_{r}-\Delta z\right) \\
& Z \Delta z=K_{z}\left(\Delta z_{r}-\Delta z\right) \\
& \left(Z+K_{z}\right) \Delta z=K_{z} \Delta z_{r} \\
\Delta z= & \left(Z+K_{z}\right)^{-1} K_{z} \Delta z_{r}=Y \Delta z_{r}
\end{aligned}
$$

with $Y=\left(Z+K_{z}\right)^{-1} K_{z}$. By backward substituting eq. (53) and (50) in eq. (47), (46), etc. we find the system solution shown in eq. (18), ..., (26).

\section{REFERENCES}

[1] Barrett Technology inc. http://www.barrett.com/robot/products-hand. htm, 2012.

[2] A. Bicchi. Force distribution in multiple whole-limb manipulation. In Proc. IEEE Int. Conf. Robotics and Automation, pages 196-201, Atlanta, 1993.

[3] A. Bicchi. On the problem of decomposing grasp and manipulation forces in multiple whole-limb manipulation. Int. Journal of Robotics and Autonomous Systems, 13:127-147, 1994.

[4] A. Bicchi. Hands for dextrous manipulation and robust grasping: a difficult road towards simplicity. IEEE Trans. on Robotics and Automation, 16(6):652-662, December 2000.

[5] A. Bicchi, M. Gabiccini, and M. Santello. Modelling natural and artificial hands with synergies. Philosophical Transactions of the Royal Society B: Biological Sciences, 366(1581):3153-3161, 2011.

[6] L. Birglen. From flapping wings to underactuated fingers and beyond: a broad look to self-adaptive mechanisms. Mechanical Sciences, 1(1):512, 2010.
[7] L. Birglen and C. M. Gosselin. Kinetostatic analysis of underactuated fingers. IEEE Transaction on Robotics and Automation, 20(2):211 221, 2004.

[8] L. Birglen and C. M. Gosselin. Grasp-state plane analysis of two-phalanx underactuated fingers. Mechanism and Machine Theory, 41(7):807 $822,2006$.

[9] L. Birglen, T Lalibertè, and C. Gosselin. Underactuated Robotic Hands, volume 40 of Springer Tracts in Advanced Robotics. Springer, 2008.

[10] C. Y. Brown and H. H. Asada. Inter-Finger Coordination and Postural Synergies in Robot Hands via Mechanical Implementation of Principal Components Analysis. In 2007 IEEE/RSJ International Conference on Intelligent Robots and System, pages 2877-2882, 2007.

[11] J. Butterfass, M. Grebenstein, H. Liu, and G. Hirzinger. DLR-hand II next generation of a dextrous robot hand. In Proc. IEEE Int. Conf. Robotics and Automation, volume 1, pages 109-114, 2001.

[12] S.F. Chen and I. Kao. Conservative congruence transformation for joint and cartesian stiffness matrices of robotic hands and fingers. The international Journal of Robotics Research, 19(9):835-847, sep 2000.

[13] S.F. Chen, Y. Li, and I. Kao. A new theory in stiffness for dextrous manipulation. In Proc. IEEE Int. Conf. on Robotics and Automation, Seoul, Korea, 2001.

[14] N. Ciblak and H. Lipkin. Asymmetric cartesian stiffness for the modeling of compliant robotic systems. In Proc. 23rd Biennial ASME Mechanisms Conference, Minneapolis, MN, 1994.

[15] M. Ciocarlie and P. Allen. A design and analysis tool for underactuated compliant hands. In Proc. IEEE/RSJ Int. Conf. on Intelligent Robots and Systems, pages 5234 -5239, oct. 2009.

[16] M. Ciocarlie, C. Goldfeder, and P. Allen. Dimensionality reduction for hand-independent dexterous robotic grasping. In Proc. IEEE/RSJ Int. Conf. on Intelligent Robots and Systems, pages 3270-3275, 2007.

[17] M. T. Ciocarlie and P. K. Allen. Hand posture subspaces for dexterous robotic grasping. The International Journal of Robotics Research, 28(7):851-867, July 2009.

[18] M.R. Cutkosky and I. Kao. Computing and controlling the compliance of a robotic hand. IEEE Transaction on Robotics and Automation, 5(2):151-165, 1989.

[19] H. de Visser and J. L. Herder. Force-directed design of a voluntary closing hand prosthesis. Journal of Rehabilitation Research \& Development, 37(3):261-271, 2000

[20] A. Dollar and R. Howe. The sdm hand: A highly adaptive compliant grasper for unstructured environments. In Experimental Robotics, pages 3-11. Springer, 2009.

[21] F urer C. Eich-Soellner E. Numerical methods in multibody dynamics. B.G. Teubner, 1998.

[22] F. Ficuciello, G. Palli, C. Melchiorri, and B. Siciliano. Experimental evaluation of postural synergies during reach to grasp with the ub hand iv. In IEEE/RSJ International Conference on Intelligent Robots and Systems (IROS), pages 1775-1780. IEEE, 2011.

[23] M. Gabiccini and A. Bicchi. On the role of hand synergies in the optimal choice of grasping forces. In Proceedings of Robotics: Science and Systems, Zaragoza, Spain, June 2010.

[24] M. Gabiccini, A. Bicchi, D. Prattichizzo, and M. Malvezzi. On the role of hand synergies in the optimal choice of grasping forces. Autonomous Robots, pages $1-18$.

[25] M. Gabiccini, M. Branchetti, and A. Bicchi. Dynamic optimization of tendon tensions in biomorphically designed hands with rolling constraints. In IEEE Int. Conf. on Robotics and Automation, pages 26982704. IEEE, 2011.

[26] G. Gioioso, G. Salvietti, M. Malvezzi, and D. Prattichizzo. An objectbased approach to map human hand synergies onto robotic hands with dissimilar kinematics. Proceedings of Robotics: Science and Systems, Sydney, Australia, 2012.

[27] C. Gosselin, F. Pelletier, and T. Laliberte. An anthropomorphic underactuated robotic hand with 15 dofs and a single actuator. In Proc. IEEE Int. Conf. Robotics and Automation, pages 749 -754, may 2008.

[28] S.C. Jacobsen, J.E. Wood, D.F. Knutti, and K.B. Biggers. The Utah/MIT dextrous hand: work in progress. The International Journal of Robotics Research, 3(4):21, 1984.

[29] T. Lalibertè and Clèment M. Gosselin. Simulation and design of underactuated mechanical hands. Mechanism and Machine Theory, 33(1-2):39 - 57, 1998.

[30] F. Lotti, P. Tiezzi, G. Vassura, L. Biagiotti, and C. Melchiorri. Ubh 3: an anthropomorphic hand with simplified endo-skeletal structure and soft continuous fingerpads. In Proceedings of the IEEE International Conference on Robotics and Automation, ICRA, volume 5, pages 47364741. IEEE, 2004. 
[31] Shadow Robot Company Ltd. Shadow hand. Retrieved from Shadowhand: http://shadowhand.com, 2009.

[32] M. Gabiccini. A twist exponential approach to gear generation with general spatial motions. MAMT, 44(2):382-400, February 2009.

[33] Gioioso G. Salvietti G. Prattichizzo D. Bicchi A. Malvezzi, M. Syngrasp: a matlab toolbox for grasp analysis of human and robotic hands. In Proceedings of the IEEE International Conference on Robotics and Automation, ICRA. IEEE, 2013. accepted for publication.

[34] M. Malvezzi and D. Prattichizzo. Evaluation of grasp stiffness in underactuated compliant hands. In Proceedings of the IEEE International Conference on Robotics and Automation, ICRA. IEEE, 2013. accepted for publication.

[35] D.J. Montana. The kinematics of contact and grasp. The International Journal of Robotics Research, 7(3):17-32, 1988.

[36] S. Mulatto, A. Formaglio, M. Malvezzi, and D. Prattichizzo. Animating a deformable hand avatar with postural synergies for haptic grasping. In Haptics: Generating and Perceiving Tangible Sensations. Eurohaptics 2010, Lecture Notes in Computer Science, pages 203-210. Springer Verlag, Amsterdam, The Netherlands, 2010.

[37] R.M. Murray, Z. Li, and S.S. Sastry. A mathematical introduction to Robotic Manipulation. 1994.

[38] L.U. Odhner and A.M. Dollar. Dexterous manipulation with underactuated elastic hands. In IEEE International Conference on Robotics and Automation (ICRA), pages 5254-5260. IEEE, 2011.

[39] E. Paljug, X. Yun, and V. Kumar. Control of rolling contacts in multiarm manipulation. Robotics and Automation, IEEE Transactions on, 10(4):441-452, 1994.

[40] A. Pashkevich, Klimchilk A., and D. Chablat. Enhanched stiffness modeling of manipulators with passive joints. Mechanism and Machine Theory, (46):662-679, 2011.

[41] D. Prattichizzo and A. Bicchi. Consistent specification of manipulation tasks for defective mechanical systems. ASME Jour. Dynam. Systems, Measurement, and Control, 119:767-777, December 1997.

[42] D. Prattichizzo and A. Bicchi. Dynamic analysis of mobility and graspability of general manipulation systems. IEEE Trans. on Robotics and Automation, 14(2):241-258, April 1998.

[43] D. Prattichizzo, M. Malvezzi, and A. Bicchi. On motion and force controllability of grasping hands with postural synergies. In Proceedings of Robotics: Science and Systems, Zaragoza, Spain, June 2010.

[44] D. Prattichizzo and J. Trinkle. Grasping. In B. Siciliano and O. Kathib, editors, Handbook on Robotics, pages 671-700. Springer, 2008.

[45] M. Santello, M. Flanders, and J. F. Soechting. Postural hand synergies for tool use. The Journal of Neuroscience, 18(23):10105-10115, December 1998

[46] M. Santello and J. F. Soechting. Force synergies for multifingered grasping. Experimental Brain Research, 133(4):457-467, August 2000.

[47] T. Wimbock, B. Jahn, and G. Hirzinger. Synergy level impedance control for multifingered hands. In International Conference on Intelligent Robots and Systems, pages 973-979. IEEE, 2011.

[48] J. Yen, E. J. Haug, and T. O. Tak. Numerical Methods for Constrained Equations of Motion in Mechanical System Dynamics. Mechanics of Structures and Machines, 19:41-76, 2010.

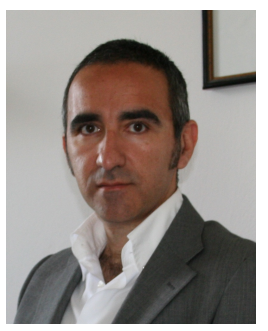

Domenico Prattichizzo M.S. degree in Electronics Engineering and the Ph.D. degree in Robotics and Automation from the University of Pisa in 1991 and 1995, respectively. Since 2002 Associate Professor of Robotics at the University of Siena. Since 2009 Scientific Consultant at Istituto Italiano di Tecnologia, Genova Italy. In 1994, Visiting Scientist at the MIT AI Lab. Co-editor of the books "Control Problems in Robotics" (2003), and "Multi-Point Physical Interaction with Real and Virtual Objects" (2005) by Springer. Guest Co-Editor of Special Issue on "Visual Servoing" of Mechatronics (2012). Guest Co-Editor of Special Issue "Robotics and Neuroscience" of the Brain Research Bulletin (2008). Coauthor of the "Grasping" chapter of "Handbook of Robotics" Springer, 2008 Since 2007 Associate Editor in Chief of the IEEE Trans. on Haptics. From 2003 to 2007, Associate Editor of the IEEE Trans. on Robotics and IEEE Trans. on Control Systems Technologies. Research interests are in haptics, grasping, visual servoing, mobile robotics and geometric control. Author of more than 200 papers in robotics and automatic control.

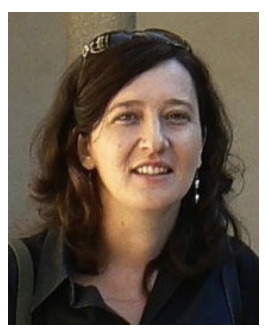

Monica Malvezzi received the Laurea Degree in Mechanical Engineering from the University of Firenze in 1999 and the Ph.D. in Mechanics and Mechanism Theory from the University of Bologna in 2003. From 2003 to 2008 she was researcher in mechanics at the University of Firenze, Energy Department, where she worked on several research project involving dynamics, localization and control of mechanisms and in particular, of vehicles. From 2008 she has been Assistant Professor of Mechanics and Mechanism Theory at the Department of Information Engineering and Mathematics of the University of Siena. Currently, her main research interests are in control of mechanical systems, robotics, multibody dynamics, haptics, grasping and dexterous manipulation.

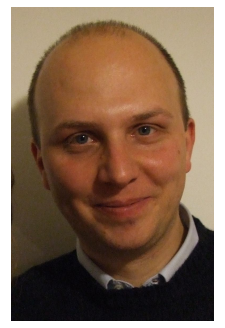

Marco Gabiccini received the Laurea degree (cum laude) and the Ph.D. both from the University of Pisa, Pisa, Italy, in 2000 and 2006, respectively. During his Ph.D. he was a visiting scholar at the GearLab, The Ohio State University, Columbus, from 2003 to 2004. Since 2001, he has been doing research at the Department of Mechanical, Nuclear and Production Engineering, University of Pisa. In 2006, he also joined the Interdepartmental Research Center E. Piaggio. He is currently a faculty member of the Department of Mechanical, Nuclear and Production Engineering (DIMNP). He teaches Robotics, Applied Mechanics and Biomechanics at the University of Pisa, Faculty of Engineering. His main research interests are in the field of theory of gearing, geometrical methods in robotics and in the areas of dynamics, kinematics and control of complex mechanical systems.

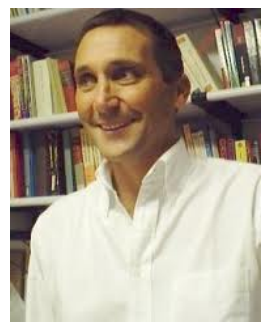

Antonio Bicchi received the Laurea degree in Mechanical Engineering from the University of Pisa in 1984, and the Doctoral degree from the University of Bologna in 1989. After a post-doctoral fellowship at the Artificial Intelligence lab, Massachusetts Institute of Technology, he joined the Faculty of Engineer ing in the University of Pisa in 1990. He is Professor of Systems Theory and Robotics in the Department of Electrical Systems and Automation (DSEA) of the University of Pisa and the Director of the Interdepartmental Research Center E. Piaggio of the University of Pisa, where he has been leading the Automation and Robotics group since 1990. His main research interests are in: dynamics, kinematics and control of complex mechanical systems, including robots, autonomous vehicles, and automotive systems; haptics and dexterous manipula tion; theory and control of nonlinear systems, in particular hybrid (logic/dynamic, symbol/signal) systems. Antonio Bicchi is a Fellow of IEEE , and elected Chair of the Conference Editorial Board of IEEE Robotics and Automation Society. 